\title{
Classical Novae at Radio Wavelengths
}

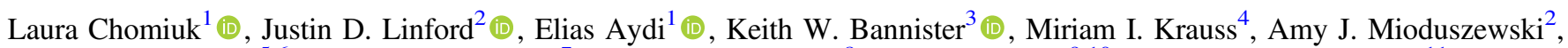 \\ Koji Mukai $^{5,6}$ (D) Thomas J. Nelson ${ }^{7}$ (D) Michael P. Rupen ${ }^{8}$, Stuart D. Ryder ${ }^{9,10}\left(\mathbb{D}\right.$, Jennifer L. Sokoloski ${ }^{11}$,

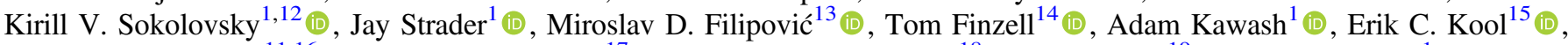 \\ Brian D. Metzger ${ }^{11,16}$ (D) , Miriam M. Nyamai ${ }^{17}$, Valério A. R. M. Ribeiro ${ }^{18}$, Nirupam Roy ${ }^{19}$ (i) , Ryan Urquhart $^{1}$ (D), and \\ Jennifer Weston ${ }^{20}$ (10 \\ ${ }^{1}$ Center for Data Intensive and Time Domain Astronomy, Department of Physics and Astronomy, Michigan State University, East Lansing, MI 48824, USA \\ chomiuk@pa.msu.edu \\ ${ }^{2}$ National Radio Astronomy Observatory, P.O. Box O, Socorro, NM 87801, USA \\ ${ }^{3}$ CSIRO Astronomy and Space Science, PO Box 76, Epping, New South Wales 1710, Australia \\ ${ }^{4}$ Pasadena City College, 1570 E. Colorado Blvd., Pasadena, CA 91106, USA \\ ${ }^{5}$ Center for Space Science and Technology, University of Maryland Baltimore County, Baltimore, MD 21250, USA \\ ${ }^{6}$ CRESST and X-ray Astrophysics Laboratory, NASA/GSFC, Greenbelt, MD 20771 USA \\ ${ }^{7}$ Department of Physics and Astronomy, University of Pittsburgh, 3941 O'Hara St., Pittsburgh, PA 15260, USA \\ ${ }^{8}$ Herzberg Institute of Astrophysics, National Research Council of Canada, Penticton, BC V2A 6J9, Canada \\ ${ }_{9}^{9}$ Department of Physics and Astronomy, Macquarie University, NSW 2109, Australia \\ ${ }^{10}$ Macquarie University Research Centre for Astronomy, Astrophysics \& Astrophotonics, Sydney, NSW 2109, Australia \\ ${ }^{11}$ Columbia Astrophysics Laboratory, Columbia University, New York, NY, USA \\ ${ }^{12}$ Sternberg Astronomical Institute, Moscow State University, Universitetskii pr. 13, 119992 Moscow, Russia \\ ${ }^{13}$ Western Sydney University, Locked Bag 1797, Penrith South DC, NSW 2751, Australia \\ ${ }^{14}$ Department of Computational Math, Science, and Engineering, Michigan State University, East Lansing, MI 48824, USA \\ ${ }^{15}$ The Oskar Klein Centre, Department of Astronomy, Stockholm University, AlbaNova, SE-106 91 Stockholm, Sweden \\ ${ }^{6}$ Center for Computational Astrophysics, Flatiron Institute, 162 W. 5th Avenue, New York, NY 10011, USA \\ ${ }^{17}$ Department of Astronomy, University of Cape Town, Private Bag X3, Rondebosch 7701, South Africa \\ ${ }^{18}$ Instituto de Telecomunicações, Campus Universitário de Santiago, 3810-193 Aveiro, Portugal \\ 19 Department of Physics, Indian Institute of Science, Bangalore 560012, India \\ ${ }^{20}$ Federated IT, 1201 Wilson Blvd, 27th Floor, Arlington, VA 22209, USA \\ Received 2021 June 29; revised 2021 August 20; accepted 2021 August 23; published 2021 December 1
}

\begin{abstract}
We present radio observations $(1-40 \mathrm{GHz})$ for 36 classical novae, representing data from over five decades compiled from the literature, telescope archives, and our own programs. Our targets display a striking diversity in their optical parameters (e.g., spanning optical fading timescales, $t_{2}=1-263$ days), and we find a similar diversity in the radio light curves. Using a brightness temperature analysis, we find that radio emission from novae is a mixture of thermal and synchrotron emission, with nonthermal emission observed at earlier times. We identify high brightness temperature emission $\left(T_{B}>5 \times 10^{4} \mathrm{~K}\right)$ as an indication of synchrotron emission in at least nine $(25 \%)$ of the novae. We find a class of synchrotron-dominated novae with mildly evolved companions, exemplified by V5589 Sgr and V392 Per, that appear to be a bridge between classical novae with dwarf companions and symbiotic binaries with giant companions. Four of the novae in our sample have two distinct radio maxima (the first dominated by synchrotron and the later by thermal emission), and in four cases the early synchrotron peak is temporally coincident with a dramatic dip in the optical light curve, hinting at a common site for particle acceleration and dust formation. We publish the light curves in a machine-readable table and encourage the use of these data by the broader community in multiwavelength studies and modeling efforts.
\end{abstract}

Unified Astronomy Thesaurus concepts: Cataclysmic variable stars (203); Novae (1127); Radio transient sources (2008); White dwarf stars (1799); Galactic radio sources (571)

Supporting material: machine-readable tables

\section{Introduction}

Classical novae are thermonuclear eruptions on the surfaces of white dwarf stars, occurring in a layer of material accreted from a binary companion (Gallagher \& Starrfield 1978; Bode \& Evans 2008). Novae are typically discovered and primarily observed as optical transient events, but novae emit detectable radiation across the entire electromagnetic spectrum, from radio to $\gamma$-ray wavelengths. The long-standing canonical picture is that the bolometric luminosity of a nova is determined by the central white dwarf, which can continue nuclear fusion in residual material on its surface for days to years after eruption, maintaining near-Eddington luminosities $\left(\sim 10^{38} \mathrm{erg} \mathrm{s}^{-1}\right)$. This bolometric luminosity originally emerges at optical wavelengths, but as the ejecta expand, the peak of the spectral energy distribution shifts blueward before settling in the extreme UV/supersoft X-ray regime (Gallagher \& Code 1974; Schwarz et al. 2011).

The recent discovery of $\mathrm{GeV} \gamma$-rays from classical novae has led to a growing appreciation for the role of shocks and nonthermal radiation in shaping the emission signatures of novae (Ackermann et al. 2014; Chomiuk et al. 2021). The relatively high $\gamma$-ray luminosities observed from novae $\left(\sim 10^{35}-10^{36} \mathrm{erg} \mathrm{s}^{-1}\right)$ imply shock luminosities that rival the Eddington luminosity of a white dwarf (Metzger et al. 2015). Signatures of shocks are also increasingly appreciated at wavelengths including (1) X-ray, where we observe relatively hard emission from hot shocked gas (Mukai et al. 2008; Gordon et al. 2021); (2) optical, where light curve features 

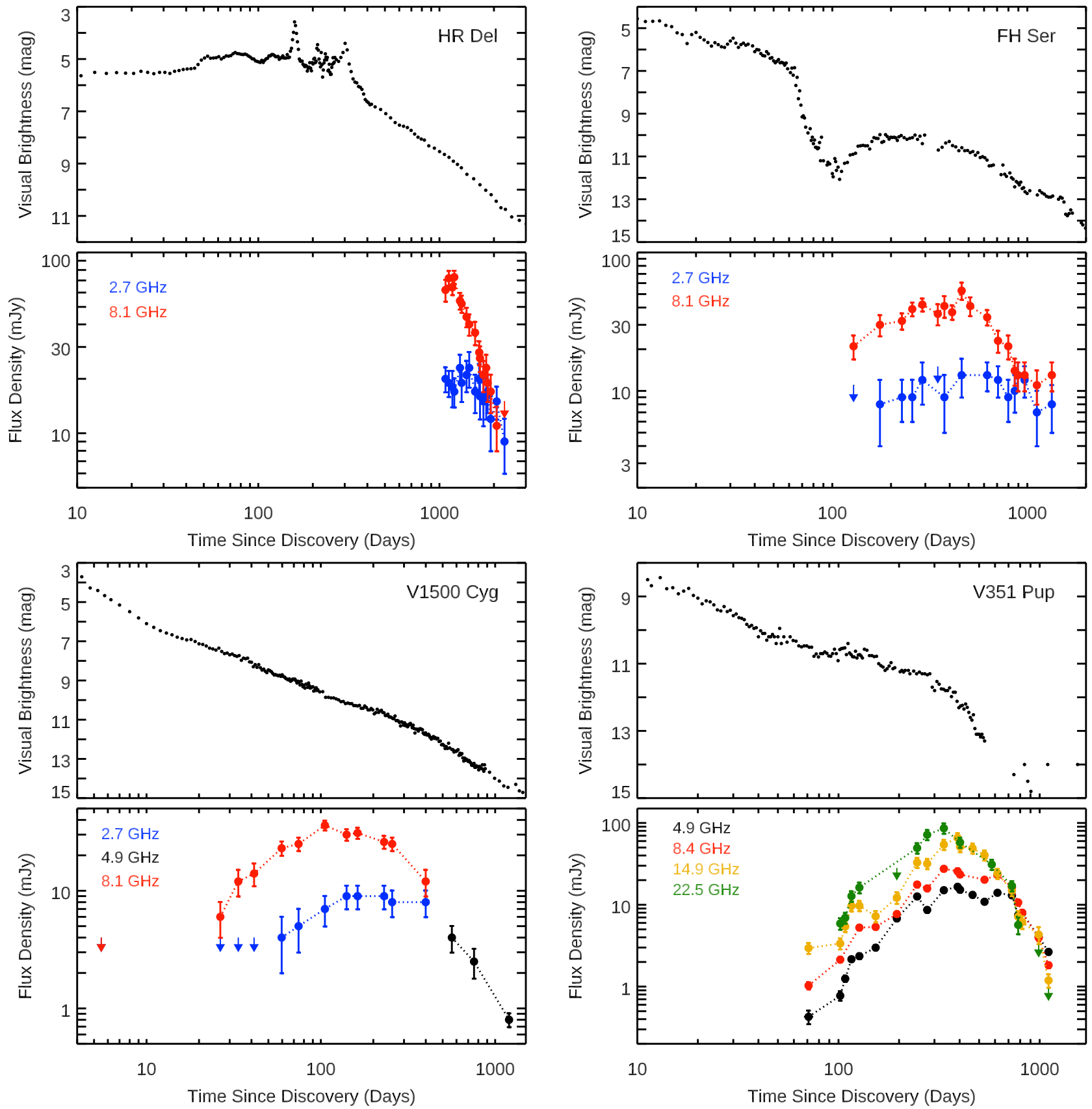

Figure 1. Previously published radio light curves, accompanied by optical light curves, for four novae (clockwise from top left): HR Del (1967), FH Ser (1970), V351 Pup (1991), and V1500 Cyg (1975). Radio epochs with nondetections are plotted with arrows. There are sporadic additional high-frequency ( $\gtrsim 15 \mathrm{GHz})$ points not plotted here. The visual-band light curves are binned AAVSO data published by Strope et al. (2010).

occur simultaneously with $\gamma$-ray features (Li et al. 2017; Aydi et al. 2020a); and (3) infrared, where shocks might lead to dust formation (Derdzinski et al. 2017).

Our understanding of radio emission from novae has also been undergoing a recent, dramatic shift. Radio observations of novae date back to the 1970s (Figure 1), when the radio signatures were interpreted as thermal free-free emission from the warm, ionized, expanding ejecta (Seaquist \& Palimaka 1977; Hjellming et al. 1979). Much as at optical wavelengths-but more simply, because line absorption/ emission should not be a significant issue-the radio luminosity at a given frequency increases until the optical depth declines to $\tau \approx 1$ within the ejecta, at which point the light curve turns over and begins to fade as the ejecta continue to drop in density. Radio light curves evolve slowly, over years, and most of these first radio observations did not commence until months after explosion. The thermal radio light curves of novae hold great promise as tracers of the ejecta mass and kinetic energy, as they should relatively directly and simply trace the ionized ejecta (Bode \& Evans 2008).

As time went on, observations of novae pushed to earlier times in the eruption, over a larger radio frequency range, and sampled more diverse eruptions. Hints that radio emission from novae may be more complex than purely thermal came with V1370 Aql and QU Vul (Figure 4; Snijders et al. 1987; Taylor et al. 1987). The high brightness temperatures $\left(>10^{5} \mathrm{~K}\right)$ of 
early emission observed in these novae indicated a role for nonthermal emission. Since 2010 and the advent of the upgraded Karl G. Jansky Very Large Array (VLA), more examples of novae with high-brightness-temperature radio emission and other evidence of synchrotron emission arrived with V1723 Aql (Krauss et al. 2011; Weston et al. 2016a; Figure 2), V5589 Sgr (Weston et al. 2016b; Figure 2), and V1324 Sco (Finzell et al. 2018; Figure 3). Hints also emerged that even the thermal emission from novae is complex, sometimes showing multiple, aspherical components (as in V959 Mon; Chomiuk et al. 2014a; Figure 3) or prolonged/ delayed ejection of material (as in T Pyx; Nelson et al. 2014; Figure 2).

It is now clear that radio emission from novae is diverse and complex, but we still do not fully understand how radio properties map to other properties of the nova eruption and host binary. Do all novae show some synchrotron emission? What determines the synchrotron luminosity and timing? Can we use thermal radio light curves to derive ejecta masses with unprecedented accuracy? How do aspherical ejecta morphology and inclination effects impact the radio light curve? These are just some of the questions we can work to answer, if we study the radio properties of novae for a large, diverse population.

Here, we compile radio observations of Galactic classical novae, foregrounding observations obtained with the VLA (both before and after its 2010 upgrade), but also including data from other telescopes like the Australia Telescope Compact Array (ATCA) and the Westerbork Synthesis Radio Telescope (WSRT). The data set represents the work of five decades, with eruptions spanning 1968-2020 and radio observations spanning 1970-2020 (Tables 1 and 2). By collecting published data, reducing archival data, and acquiring new data, we present radio light curves for 36 novae (24 with new data presented here) and include poorer-quality data for seven more objects in the Appendix (mostly nondetections). In Section 2, we describe the nova sample and the data acquisition and reduction. In Section 3, we analyze these light curves by considering not only radio flux density but also luminosity and brightness temperature. We consider the relative roles of thermal and nonthermal emission in the radio light curves and conclude in Section 4.

\section{Data}

\subsection{Sample}

We worked to collect all radio light curves for novae, including both published data sets and archival unpublished VLA data. We searched the VLA archive for all novae that erupted since 1980, using the Galactic nova catalogs curated by the Central Bureau for Astronomical Telegrams ${ }^{21}$ and $\mathrm{K}$. Mukai. $^{22}$ Most of the recent VLA observations (after 2006) were obtained by our ENova collaboration, and we also include a few novae that our collaboration targeted with ATCA. The sample of novae with sufficient radio data to enable light-curve construction, including those already published, is listed in Tables 1 and 2. Their diverse multiwavelength properties are cataloged and discussed in Table 1 and Section 2.4. Novae are selected for radio campaigns for a multitude of reasons,

\footnotetext{
21 http://www.cbat.eps.harvard.edu/nova_list.html

22 https://asd.gsfc.nasa.gov/Koji.Mukai/novae/novae.html
}

including high optical brightness, $\mathrm{GeV} \gamma$-ray detection, or recurrent nova status.

We do not consider recurrent novae (those observed in human history to have more than one nova explosion) as a unique subclass but instead consider them as the extreme end of a continuum of recurrence times; all novae should recur if one waits long enough. We therefore include all novae with main-sequence or subgiant companions, whether they are "recurrent" or "classical" (only one eruption observed thus far). We exclude V445 Pup, as it is the only known helium nova and has a distinct synchrotron-dominated radio light curve (Nyamai et al. 2021). We also exclude novae suspected to have more evolved red giant companions but encourage a future study of embedded novae like RS Oph, V745 Sco, V407 Cyg, V1534 Sco, V1535 Sco, and V3890 Sgr, keeping in mind that in some of these systems the orbital period is not yet known. We do include V392 Per here, which has a binary orbital period of 3.4 days, indicative of a companion that has just begun its ascent up the red giant branch and has luminosity class III/IV (Munari et al. 2020). As Munari et al. point out, V392 Per therefore serves as a useful touchstone bridging novae in cataclysmic variables and novae in symbiotic systems, and we include it here for this reason.

Radio observation campaigns often last years for a single nova, and over half a century, observation campaigns start and terminate for a range of reasons. The ideal case is where observations begin early in the eruption, bridge the rise from nondetections to luminous emission, and then continue as the nova fades back to nondetections. The number of such thorough campaigns has been increasing, but many of the radio observations presented in this paper fall short of this ideal. Campaigns may start too late and miss interesting early structure in the radio light curve. Campaigns may terminate too early-because of scheduling difficulties on telescopes or even changes in employment or telescope technology — and miss the evolution of the thermal maximum.

There are also novae that have a few radio observations, but the data are not sufficient to really constrain the light-curve evolution. For completeness, we have sought to collect these observations in the Appendix, but for clarity we do not include them in the main discussions of this paper. The observation campaigns relegated to the Appendix tend to be (1) only nondetections; or (2) only one or two epochs featuring detections that are consistent with thermal expansion.

In Figures 1-3, we plot the previously published radio light curves and place them in context of each nova's optical light curve. Figures 4-9 plot light curves for 24 novae with new radio observations; the novae are in chronological order. This sample is a mix of older archival data collected by diverse nova researchers and newer data collected by our team.

\subsection{Radio Data Reduction}

For data obtained with the historic VLA (1980-2009), all data were obtained in continuum mode, with two intermediate frequencies each $50 \mathrm{MHz}$ wide. Detailed information about the observations, including calibrators observed, is available in the NRAO archive. ${ }^{23}$ Data were typically obtained at the $C$ (4.9 $\mathrm{GHz}), X(8.4 \mathrm{GHz}), U(14.9 \mathrm{GHz})$, and $K(22.4 \mathrm{GHz})$ bands, with occasional observations at the $L$ band $(1.4 \mathrm{GHz}$; novae are usually faintest at low frequencies because their emission is

\footnotetext{
23 https://archive.nrao.edu/archive/advquery.jsp
} 
Table 1

Properties of Radio-observed Novae (in Chronological Order of Discovery)

\begin{tabular}{|c|c|c|c|c|c|c|c|c|c|c|}
\hline Name & $\begin{array}{l}\text { R.A. (J2000) } \\
\text { (h:m:s) }\end{array}$ & $\begin{array}{l}\text { Decl. (J2000) } \\
\left.\text { (d: }::^{\prime \prime}\right)\end{array}$ & $\begin{array}{c}\text { Discovery Date } \\
\text { (UT) }\end{array}$ & $\begin{array}{l}V_{\text {peak }} \\
(\mathrm{mag})\end{array}$ & $\begin{array}{c}t_{2} \\
\text { (days) }\end{array}$ & $\begin{array}{c}E(B-V) \\
(\mathrm{mag})\end{array}$ & $\begin{array}{c}v_{1} \\
\left(\mathrm{~km} \mathrm{~s}^{-1}\right)\end{array}$ & $\begin{array}{c}v_{2} \\
\left(\mathrm{~km} \mathrm{~s}^{-1}\right)\end{array}$ & $\begin{array}{l}\text { Distance }^{\mathrm{b}} \\
(\mathrm{kpc})\end{array}$ & $\begin{array}{l}P_{\text {orb }}{ }^{\mathrm{c}} \\
(\mathrm{hr})\end{array}$ \\
\hline HR Del & 204220.344 & +190939.16 & $1967 \mathrm{Jul} 8^{\mathrm{h}}$ & $3.6^{\mathrm{e}}$ & $167^{\mathrm{a}}$ & $0.17 \pm 0.02^{\mathrm{f}}$ & $800^{\mathrm{i}}$ & $1300^{\mathrm{i}}$ & $0.87 \pm 0.02$ & $5.1^{\mathrm{g}}$ \\
\hline FH Ser & 183047.042 & +023651.98 & $1970 \mathrm{Feb} 13^{\mathrm{j}}$ & $4.5^{\mathrm{e}}$ & $49^{\mathrm{e}}$ & $0.6 \pm 0.1^{\mathrm{f}}$ & $800^{\mathrm{k}}$ & $1750^{\mathrm{k}}$ & $1.00_{-0.06}^{+0.07}$ & $\mathrm{MS}^{1}$ \\
\hline V1500 Cyg & 211136.571 & +480901.86 & 1975 Aug $29^{\mathrm{m}}$ & $1.9^{\mathrm{e}}$ & $2^{\mathrm{e}}$ & $0.45 \pm 0.07^{\mathrm{f}}$ & $?$ & $4000^{\mathrm{n}}$ & $1.71_{-0.28}^{+0.40}$ & $3.4^{\mathrm{g}}$ \\
\hline V1370 Aql & 192321.241 & +022926.20 & $1982 \operatorname{Jan} 27^{\circ}$ & $7.7^{\mathrm{e}}$ & $15^{\mathrm{e}}$ & $0.35 \pm 0.05^{\mathrm{f}}$ & $?$ & $2200^{\mathrm{p}}$ & $2.8_{-0.8}^{+1.7}$ & $\mathrm{MS}^{\mathrm{q}}$ \\
\hline PW Vul & 192605.050 & +272158.10 & $1984 \mathrm{Jul} 28^{\mathrm{r}}$ & $6.4^{\mathrm{e}}$ & $44^{\mathrm{e}}$ & $0.6 \pm 0.1^{\mathrm{f}}$ & $800^{\mathrm{s}}$ & $1650^{\mathrm{s}}$ & $2.16_{-0.32}^{+0.45}$ & $5.1^{\mathrm{g}}$ \\
\hline QU Vul & 202646.021 & +275043.11 & $1984 \operatorname{Dec} 22^{\mathrm{t}}$ & $5.3^{\mathrm{e}}$ & $20^{\mathrm{e}}$ & $0.55 \pm 0.05^{\mathrm{f}}$ & $700^{\mathrm{u}}$ & $1400^{\mathrm{u}}$ & $1.4_{-0.4}^{1.1}$ & $2.7^{\mathrm{g}}$ \\
\hline V1819 Cyg & 195437.545 & +354215.40 & $1986 \operatorname{Aug} 4^{v}$ & $9.3^{\mathrm{e}}$ & $95^{\mathrm{e}}$ & $0.35 \pm 0.15^{\mathrm{f}}$ & $650^{\mathrm{w}}$ & $1450^{\mathrm{w}}$ & $7.4 \pm 1.4^{\mathrm{f}}$ & $?$ \\
\hline V827 Her & 184342.506 & +151918.22 & $1987 \operatorname{Jan} 25^{\mathrm{x}}$ & $7.5^{\mathrm{e}}$ & $21^{\mathrm{e}}$ & $0.1^{\mathrm{f}}$ & $?$ & $2000^{y}$ & $2.1_{-0.7}^{+1.4}$ & $?$ \\
\hline V838 Her & 184631.468 & +121402.00 & $1991 \operatorname{Mar} 24^{z}$ & $5.3^{\mathrm{e}}$ & $1^{\mathrm{e}}$ & $0.5 \pm 0.1^{\mathrm{f}}$ & $?$ & $4500^{\mathrm{aa}}$ & $3.2_{-1.6}^{+2.5}$ & $7.1^{\mathrm{g}}$ \\
\hline V351 Pup & $08 \quad 1138.391$ & -350730.27 & 1991 Dec $27^{\mathrm{ab}}$ & $6.4^{\mathrm{e}}$ & $9^{\mathrm{e}}$ & $0.5 \pm 0.1^{\mathrm{f}}$ & $?$ & $3000^{\mathrm{ac}}$ & $5 \pm 1.5^{\mathrm{ad}}$ & $2.8^{\mathrm{g}}$ \\
\hline V1974 Cyg & 203031.651 & +523750.74 & $1992 \mathrm{Feb} 19^{\mathrm{ae}}$ & $4.3^{\mathrm{e}}$ & $19^{\mathrm{e}}$ & $0.26 \pm 0.03^{\mathrm{f}}$ & $1350^{\mathrm{af}}$ & $2300^{\mathrm{af}}$ & $1.58_{-0.13}^{+0.14}$ & $2.0^{\mathrm{g}}$ \\
\hline V705 Cas & 234147.230 & +573100.79 & 1993 Dec $7^{\text {ag }}$ & $5.7^{\mathrm{e}}$ & $33^{\mathrm{e}}$ & $0.41 \pm 0.06^{\mathrm{f}}$ & $?$ & $1700^{\mathrm{ah}}$ & $2.44_{-0.33}^{+0.44}$ & $5.5^{\mathrm{g}}$ \\
\hline V1419 Aql & 191306.791 & +013423.23 & 1993 May $14^{\text {ai }}$ & $7.6^{\mathrm{e}}$ & $25^{\mathrm{e}}$ & $0.50 \pm 0.05^{\mathrm{f}}$ & $900^{\mathrm{aj}}$ & $1400^{\mathrm{aj}}$ & $5.0_{-2.6}^{+4.0}$ & $?$ \\
\hline V723 Cas & 010505.354 & +540040.23 & 1995 Aug $24^{\mathrm{ak}}$ & $7.1^{\mathrm{e}}$ & $263^{e}$ & $0.45^{\mathrm{f}}$ & $?$ & $1750^{\mathrm{al}}$ & $4.6_{-0.6}^{+0.8}$ & $16.6^{\mathrm{g}}$ \\
\hline U Sco & 162230.779 & -175243.29 & $1999 \mathrm{Feb} 24^{\mathrm{am}}$ & $7.5^{\mathrm{e}}$ & $1^{\mathrm{e}}$ & $0.14 \pm 0.12^{\mathrm{f}}$ & $?$ & $5000^{\text {an }}$ & $12 \pm 2^{\text {ao }}$ & $29.5^{\mathrm{g}}$ \\
\hline V4743 Sgr & 190109.339 & -220006.12 & $2002 \operatorname{Sep} 20^{\mathrm{ap}}$ & $5.0^{\mathrm{e}}$ & $6^{\mathrm{e}}$ & $0.25^{\mathrm{f}}$ & $?$ & $2700^{\mathrm{aq}}$ & $3.7_{-0.7}^{+0.9}$ & $6.7^{\mathrm{g}}$ \\
\hline V598 Pup & 070542.501 & $-38 \quad 1439.32$ & 2007 Jun $5^{\text {ar }}$ & $4^{\mathrm{ar}}$ & $9^{\mathrm{ar}}$ & $0.09 \pm 0.08^{\mathrm{f}}$ & $?$ & $2100^{\mathrm{ar}}$ & $1.7 \pm 0.1$ & $?$ \\
\hline V2491 Cyg & 194301.973 & +321913.46 & 2008 Apr $10^{\text {as }}$ & $7.5^{\mathrm{e}}$ & $4^{\mathrm{e}}$ & $0.23 \pm 0.01^{\mathrm{f}}$ & $?$ & $4500^{\text {at }}$ & $7.8_{-2.3}^{+3.5}$ & $2.6^{\mathrm{g}}$ \\
\hline V2672 Oph & $1738 \quad 19.710$ & -264413.58 & 2009 Aug $16^{\text {au }}$ & $11.4^{\mathrm{au}}$ & $2^{\mathrm{av}}$ & $1.6 \pm 0.1^{\mathrm{f}}$ & $?$ & $5000^{\text {av }}$ & $9.4_{-4.2}^{+6.7}$ & $\mathrm{MS}$ or $\mathrm{SG}^{\mathrm{av}}$ \\
\hline V1723 Aql & $184738.38^{\mathrm{a}}$ & $-0347 \quad 14.1$ & 2010 Sep $11^{\text {aw }}$ & $16.0^{\mathrm{ax}}$ & $12^{\mathrm{ax}}$ & $4.3^{\mathrm{d}}$ & $?$ & $1500^{\text {aw }}$ & $5.7 \pm 0.4^{\text {ay }}$ & $?$ \\
\hline T Pyx & 090441.503 & $-32 \quad 2247.50$ & 2011 Apr $14^{\mathrm{az}}$ & $6.4^{\mathrm{e}}$ & $32^{\mathrm{e}}$ & $0.25 \pm 0.02^{\mathrm{e}}$ & $?$ & $2000^{\text {ba }}$ & $2.60_{-0.18}^{+0.20}$ & $1.8^{\mathrm{bb}}$ \\
\hline V5589 Sgr & 174528.033 & -230522.80 & 2012 Apr $21^{\mathrm{ca}}$ & $8.8^{\mathrm{f}}$ & $4.5^{\mathrm{f}}$ & $0.8 \pm 0.2^{\mathrm{f}}$ & $?$ & $4800^{\text {bd }}$ & $7.7_{-3.2}^{+5.10}$ & $38.2^{\mathrm{bc}}$ \\
\hline V1324 Sco & $175053.94^{\mathrm{a}}$ & -323720.5 & 2012 Jun $1^{\text {be }}$ & $10.1^{\mathrm{f}}$ & $25^{\mathrm{f}}$ & $1.2 \pm 0.1^{\mathrm{f}}$ & 1300 & 3200 & $>6.5^{\mathrm{bf}}$ & $3.2^{\mathrm{g}}$ \\
\hline V959 Mon & 063938.600 & +055352.84 & 2012 Jun $19^{\text {bg }}$ & $?$ & $?$ & $0.4 \pm 0.1^{\mathrm{f}}$ & 2100 & $?$ & $1.4 \pm 0.4^{\mathrm{bh}}$ & $7.1^{\mathrm{bi}}$ \\
\hline V809 Cep & 230804.722 & +604651.75 & $2013 \mathrm{Feb}^{\mathrm{ad}}$ & $11.2^{\mathrm{bj}}$ & $16^{\mathrm{bj}}$ & $1.7^{\mathrm{bj}}$ & 800 & 1600 & $>6.0^{\mathrm{bj}}$ & MS or $S^{b j}$ \\
\hline V339 Del & 202330.682 & +204603.64 & 2013 Aug 14 & $4.4^{\mathrm{bk}}$ & $10^{\mathrm{bk}}$ & $0.18 \pm 0.04^{\mathrm{bk}}$ & 1000 & 2600 & $4.9 \pm 1.0^{\mathrm{bl}}$ & $3.1^{\mathrm{bm}}$ \\
\hline V1369 Cen & 135445.323 & -590904.30 & 2013 Dec 2 & $3.3^{\mathrm{bl}}$ & $40^{\mathrm{bl}}$ & $0.06 \pm 0.01^{\mathrm{bl}}$ & 1150 & 1700 & $1.0 \pm 0.1^{\mathrm{bl}}$ & $3.8^{\mathrm{bn}}$ \\
\hline V5666 Sgr & 182508.769 & -223603.12 & 2014 Jan $26^{\text {bo }}$ & $8.7^{\mathrm{d}}$ & $90^{\mathrm{d}}$ & ? & 700 & 1200 & $6.1_{-1.5}^{+2.4}$ & $?$ \\
\hline V2659 Cyg & 202142.321 & +310329.29 & $2014 \mathrm{Mar} 30^{\mathrm{bp}}$ & $10.9^{\mathrm{d}}$ & $115^{\mathrm{d}}$ & $0.63^{\mathrm{bq}}$ & 700 & 1300 & $6.8_{-1.7}^{+2.7}$ & $?$ \\
\hline V5667 Sgr & 181425.159 & -255434.57 & $2015 \mathrm{Feb} 12^{\mathrm{br}}$ & $9.0^{\mathrm{d}}$ & $55^{\mathrm{d}}$ & $?$ & 1500 & $?$ & $5.6_{-1.0}^{+1.5}$ & $?$ \\
\hline V5668 Sgr & $183656.84^{\mathrm{a}}$ & -285540.1 & 2015 Mar $15^{\mathrm{bs}}$ & $4.3^{\mathrm{bt}}$ & $100^{\mathrm{bt}}$ & $0.7 \pm 0.1^{\mathrm{bl}}$ & 1100 & 1600 & $2.8 \pm 0.5^{\mathrm{bl}}$ & $?$ \\
\hline V5855 Sgr & $181028.29^{\mathrm{a}}$ & -272959.4 & 2016 Oct $20^{\text {bu }}$ & $7.5^{\mathrm{f}}$ & $18^{\mathrm{f}}$ & $0.5^{\mathrm{f}}$ & 800 & 2700 & $3.9 \pm 0.5$ & $?$ \\
\hline V5856 Sgr & $182052.25^{\mathrm{a}}$ & $\begin{array}{lll}-28 & 22 & 12.2\end{array}$ & 2016 Oct $25^{\text {bv }}$ & $5.9^{\mathrm{f}}$ & $11^{\mathrm{f}}$ & $1.02^{\mathrm{f}}$ & 900 & 2800 & $2.5 \pm 0.5^{\mathrm{bl}}$ & $?$ \\
\hline V357 Mus & 112615.003 & -653124.21 & 2018 Jan $14^{\mathrm{bw}}$ & $7.0^{\mathrm{bl}}$ & $40^{\mathrm{bl}}$ & $0.5 \pm 0.1^{\mathrm{bl}}$ & 750 & 2500 & $3.3_{-0.7}^{+1.2}$ & $?$ \\
\hline V906 Car & 103615.413 & -593553.64 & $2018 \operatorname{Mar} 16^{\mathrm{bx}}$ & $5.9^{\mathrm{bx}}$ & $44^{\mathrm{bx}}$ & $0.35 \pm 0.05^{\mathrm{bx}}$ & 350 & 2500 & $4 \pm 1.5^{\mathrm{bx}}$ & $1.6 / 3.3^{\mathrm{bx}}$ \\
\hline V392 Per & 045321.370 & +472125.84 & 2018 Apr $29^{\text {by }}$ & $5.6^{\mathrm{bl}}$ & $3^{\mathrm{bl}}$ & $0.40 \pm 0.05^{\mathrm{bl}}$ & 2800 & 4100 & $3.5_{-0.5}^{+0.7}$ & $81.9^{\mathrm{bz}}$ \\
\hline
\end{tabular}

Notes.

${ }^{\text {a }}$ Position was measured using VLA radio data presented here.

${ }^{\mathrm{b}}$ Distances are determined from Gaia EDR3 parallaxes, unless otherwise noted.

${ }^{c}$ In some cases, the orbital period is not known, but the nature of the companion can be determined as main sequence (MS), subgiant (SG), or red giant (RG).

${ }^{\mathrm{d}}$ This work. ${ }^{\mathrm{e}}$ Strope et al. (2010). ${ }^{\mathrm{f}}$ Özdönmez et al. (2018). ${ }^{\mathrm{g}}$ Ritter \& Kolb (2003). ${ }^{\mathrm{h}}$ Candy et al. (1967). ${ }^{\mathrm{i}}$ Hutchings (1970). ${ }^{\mathrm{j}}$ Seki et al. (1970).

${ }^{\mathrm{k}}$ Rosino et al. (1986). ${ }^{\mathrm{l}}$ Darnley et al. (2012). ${ }^{\mathrm{m}}$ Honda et al. (1975). ${ }^{\mathrm{n}}$ Hutchings et al. (1978). ${ }^{\mathrm{o}}$ Kosai et al. (1982). ${ }^{\mathrm{p}}$ Rosino et al. (1983).

${ }^{\mathrm{q}}$ Tappert et al. (2014). ${ }^{\mathrm{r}}$ Kosai et al. (1984). ${ }^{\mathrm{s}}$ Rosino \& Iijima (1987). ${ }^{\mathrm{t}}$ Collins et al. (1984). ${ }^{\mathrm{u}}$ Rosino et al. (1992). ${ }^{\mathrm{v}}$ Wakuda \& Huruhata (1986).

${ }^{\mathrm{w}}$ Andrillat \& Houziaux (1989). ${ }^{\mathrm{x}}$ Kosai et al. (1987). ${ }^{\mathrm{y}}$ Andrillat \& Houziaux (1987). ${ }^{\mathrm{z}}$ Sugano et al. (1991). ${ }^{\text {aa }}$ Iijima \& Cassatella (2010).

${ }^{a b}$ Camilleri et al. (1992). ${ }^{\text {ac }}$ della Valle et al. (1992). ${ }^{\text {ad }}$ Wendeln et al. (2017). ${ }^{\text {ae }}$ Collins et al. (1992). ${ }^{\text {af }}$ Chochol et al. (1993). ${ }^{\text {ag }}$ Nakano et al. (1993).

${ }^{\text {ah }}$ Hauschildt et al. (1994). ${ }^{\text {ai }}$ Hirayama et al. (1993). ${ }^{\text {aj }}$ Arkhipova et al. (1994). ${ }^{\text {ak }}$ Hirosawa et al. (1995). ${ }^{\text {al }}$ Iijima (2006). ${ }^{\text {am }}$ Schmeer et al. (1999).

${ }^{\text {an }}$ Anupama et al. (2013). ${ }^{\text {ao }}$ Schaefer (2010). ${ }^{\text {ap }}$ Kato et al. (2002). ${ }^{\text {aq }}$ Morgan et al. (2003). ${ }^{\text {ar }}$ Read et al. (2008). ${ }^{\text {as }}$ Nakano et al. (2008).

${ }^{a t}$ Munari et al. (2011b). ${ }^{\text {au }}$ Nakano et al. (2009). ${ }^{\text {av }}$ Munari et al. (2011a). aw Yamanaka et al. (2010) and Balam et al. (2010). ${ }^{\text {ax }}$ Nagashima et al. (2013).

${ }^{\text {ay }}$ Weston et al. (2016a). ${ }^{\text {az }}$ Waagan et al. (2011). ${ }^{\text {ba }}$ Pavana et al. (2019). ${ }^{\text {bb }}$ Uthas et al. (2010). ${ }^{\text {bc }}$ Mróz et al. (2015). ${ }^{\text {bd }}$ Weston et al. (2016b).

be Finzell et al. (2018). ${ }^{\text {bf }}$ Finzell et al. (2015). ${ }^{\text {bg }}$ Ackermann et al. (2014). ${ }^{\text {bh }}$ Linford et al. (2015). ${ }^{b i}$ Page et al. (2013). ${ }^{\text {bj }}$ Munari et al. (2014).

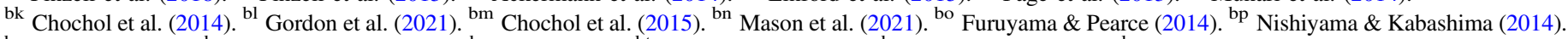

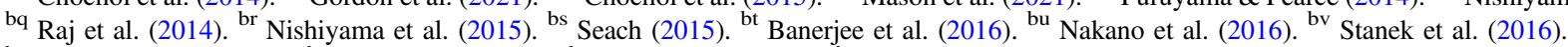

${ }^{\text {bw }}$ Kaufman et al. (2018). ${ }^{\text {bx }}$ Aydi et al. (2020a). ${ }^{\text {by }}$ Endoh et al. (2018). ${ }^{\text {bz }}$ Munari et al. (2020). ${ }^{\text {ca }}$ Korotkiy et al. (2012).

(This table is available in machine-readable form.) 
Table 2

Overview of Radio Observations of Novae

\begin{tabular}{|c|c|c|c|c|c|c|}
\hline Name & Telescope & Date Range & $\begin{array}{l}t_{\max }{ }^{d} \\
\text { (days) }\end{array}$ & $\begin{array}{l}S_{\max }^{\mathrm{d}} \\
(\mathrm{mJy})\end{array}$ & Figure & Reference \\
\hline HR Del & Combination $^{\mathrm{a}}$ & 1970 Jun 22-1978 Dec 12 & $1208^{\mathrm{e}}$ & $73^{\mathrm{e}}$ & 1 & Hjellming et al. (1979) \\
\hline FH Ser & Combination $^{\mathrm{b}}$ & 1970 Jun 22-1978 Dec 12 & $460^{\mathrm{e}}$ & $53^{\mathrm{e}}$ & 1 & Hjellming et al. (1979) \\
\hline V1500 Cyg & Combination $^{\mathrm{c}}$ & 1975 Aug 31-1978 Dec 12 & $105^{\mathrm{e}}$ & $36^{\mathrm{e}}$ & 1 & Hjellming et al. (1979) and Seaquist et al. (1980) \\
\hline V1370 Aql & WSRT/VLA & 1982 Mar 27-1987 Jan 24 & $\leqslant 142^{\mathrm{f}}$ & $\geqslant 14.2^{\mathrm{f}}$ & 4 & Snijders et al. (1987); Bode et al. (1987) and this work \\
\hline PW Vul & VLA & 1984 Aug 17-1987 Nov 28 & 600.6 & 2.85 & 4 & This work \\
\hline QU Vul & VLA & 1985 Jul 16-1988 Oct 24 & 690.0 & 14.35 & 4 & Taylor et al. (1987), Taylor et al. (1988) and this work \\
\hline V1819 Cyg & VLA & 1987 Jan 17-1991 May 31 & 556.5 & 0.88 & 4 & This work \\
\hline V827 Her & VLA & 1987 Jul 2-1989 Feb 14 & 307.1 & 2.02 & 5 & This work \\
\hline V838 Her & VLA & 1991 Mar 28-1991 Oct 31 & 25.5 & 4.8 & 5 & This work \\
\hline V351 Pup & VLA & 1992 Mar 7-1995 Jan 7 & 391.0 & 16.6 & 1 & Wendeln et al. (2017) \\
\hline V1974 Cyg & VLA & 1992 Feb 25-1996 Feb 6 & 344.6 & 24.59 & 5 & Hjellming (1996) and this work \\
\hline V705 Cas & VLA/Merlin & 1993 Dec 24-1998 Dec 8 & 108.5 & 3.37 & 5 & Eyres et al. (2000) and this work \\
\hline V1419 Aql & VLA & 1993 Jun 19-1995 Jul 5 & 214.8 & 0.83 & 6 & This work \\
\hline V723 Cas & Merlin & 1996 Dec $13-2001$ Oct 26 & 1697.5 & 13.5 & 2 & Heywood et al. (2005) \\
\hline U Sco (1987) & VLA & 1987 Jun 2-1987 Jul 5 & & & & This work \\
\hline U Sco (1999) & VLA & 1999 Mar 4-1999 Apr 25 & $33.4^{\mathrm{e}}$ & $0.14^{\mathrm{e}}$ & 6 & This work \\
\hline U Sco (2010) & GMRT & 2010 Jan 29-2010 Mar 2 & & & & Anupama et al. (2013) \\
\hline V4743 Sgr & VLA & 2002 Oct 11-2003 Feb 21 & $\geqslant 154.6^{\mathrm{e}}$ & $\geqslant 5.10^{\mathrm{e}}$ & 6 & This work \\
\hline V598 Pup & VLA & 2007 Nov 18-2008 Aug 3 & $205.0^{\mathrm{e}}$ & $11.1^{\mathrm{e}}$ & 6 & This work \\
\hline V2491 Cyg & VLA & 2008 Apr 28-2008 Sep 19 & $41.4^{\mathrm{e}}$ & $1.46^{\mathrm{e}}$ & 7 & This work \\
\hline V2672 Oph & VLA & 2009 Sep $1-2009$ Nov 7 & $\leqslant 16.1^{\mathrm{e}}$ & $\geqslant 0.55^{\mathrm{e}}$ & 7 & This work \\
\hline V1723 Aql & Jansky VLA & 2010 Sep 25-2014 Mar 15 & 57.3 & 4.08 & 2 & Krauss et al. (2011) and Weston et al. (2016a) \\
\hline T Pyx & Jansky VLA & 2011 Apr 22-2012 Sep 23 & 265.9 & 4.35 & 2 & Nelson et al. (2014) \\
\hline V5589 Sgr & Jansky VLA & 2012 Apr 23-2013 Aug 26 & 62.3 & 3.42 & 2 & Weston et al. (2016b) \\
\hline V1324 Sco & Jansky VLA & 2012 Jun 26-2014 Dec 18 & 72.2 & 1.39 & 3 & Finzell et al. (2018) \\
\hline V959 Mon & Jansky VLA & 2012 Jun 30-2014 Feb 25 & 157.3 & 25.94 & 3 & Chomiuk et al. (2014a) \\
\hline V809 Cep & Jansky VLA & 2013 Feb 14-2016 Jan 28 & 581.2 & 0.53 & 3 & Babul et al. (2021) \\
\hline V339 Del & Jansky VLA & 2013 Aug 16-2017 Jul 8 & 612.7 & 1.93 & 3 & M. M. Nyamai et al. (in preparation) \\
\hline V1369 Cen & ATCA & 2013 Dec 5-2014 Apr 1 & $\geqslant 176.0^{\mathrm{e}}$ & $\geqslant 29.9^{\mathrm{e}}$ & 7 & This work \\
\hline V5666 Sgr & Jansky VLA & 2014 Feb 19-2017 Aug 22 & 929.6 & 0.34 & 7 & This work \\
\hline V2659 Cyg & Jansky VLA & 2014 Apr 5-2018 Sep 27 & 1024.6 & 0.97 & 8 & This work \\
\hline V5667 Sgr & Jansky VLA & 2015 Feb 19-2019 Dec 22 & 1307.0 & 1.62 & 8 & This work \\
\hline V5668 Sgr & Jansky VLA & 2015 Mar 17-2019 Dec 22 & 385.5 & 15.51 & 8 & This work \\
\hline V5855 Sgr & Jansky VLA & 2016 Nov 4-2021 Feb 9 & 692.0 & 0.62 & 8 & This work \\
\hline V5856 Sgr & Jansky VLA & 2016 Nov 11-2021 Feb 9 & 1148.8 & 2.36 & 9 & This work \\
\hline V357 Mus & ATCA & 2018 Jan 18-2020 Sep 12 & 64.0 & 31.0 & 9 & This work \\
\hline V906 Car & ATCA & 2018 Apr 3-2020 Sep 12 & 911.0 & 11.5 & 9 & This work \\
\hline V392 Per & Jansky VLA & 2018 Apr 30-2020 May 15 & 32.6 & 5.23 & 9 & This work \\
\hline
\end{tabular}

Notes.

${ }^{a}$ NRAO Interferometer/NRAO $11 \mathrm{~m} /$ NRAO $140 \mathrm{ft} /$ Bonn $100 \mathrm{~m} / \mathrm{VLA}$.

b NRAO Interferometer/NRAO $11 \mathrm{~m} / \mathrm{NRAO} 140 \mathrm{ft} / \mathrm{VLA}$.

${ }^{\mathrm{c}}$ WSRT/NRAO Interferometer/ARO $46 \mathrm{~m} / \mathrm{NRAO} 11 \mathrm{~m} / \mathrm{VLA}$.

d Measured at $C$ band (4-6 GHz) unless it is not available and otherwise noted in this column.

e Measured at $X$ band (8-9 GHz).

${ }^{\mathrm{f}}$ Measured at $1.5 \mathrm{GHz}$.

$\mathrm{g}$ This column points to the relevant optical/radio light-curve figure (see Figures 1-9).

(This table is available in machine-readable form.)

predominantly thermal). Data were edited, calibrated, and imaged using standard routines in AIPS (Greisen 2003). When possible, we estimated the flux density of the complex gain calibrator by a bootstrapping comparison with a standard absolute flux calibrator (e.g., 3C286). However, many observations did not include an observation of an absolute flux calibrator, and in these cases we are forced to assume a value for the complex gain calibrator, taking the flux estimates determined in adjacent epochs with full calibration.

Observations with the Karl G. Jansky VLA (2010-present) yield data with substantially larger bandwidths and volumes. Observations were typically obtained in the $C(4-8 \mathrm{GHz}), K u$
(12-18 GHz), and $K a(26.5-40 \mathrm{GHz})$ bands and sometimes in the $L$ band (1-2 GHz). During the first few years of the Jansky VLA (i.e., 2010-2013), data were obtained with 8 bit samplers, which yielded two chunks of $1 \mathrm{GHz}$ bandwidth, which can be flexibly placed and sampled with $2 \mathrm{MHz}$ wide channels. Later, we transitioned to using 3 bit samplers, which can yield up to $8 \mathrm{GHz}$ of bandwidth. All observations were obtained in full Stokes mode, although polarization calibration was not typically carried out. As can be seen in Table 3, sometimes observing frequencies drift over a monitoring campaign, due to efforts to avoid radio-frequency interference (RFI) or changes in instrumental capabilities. Jansky VLA data 

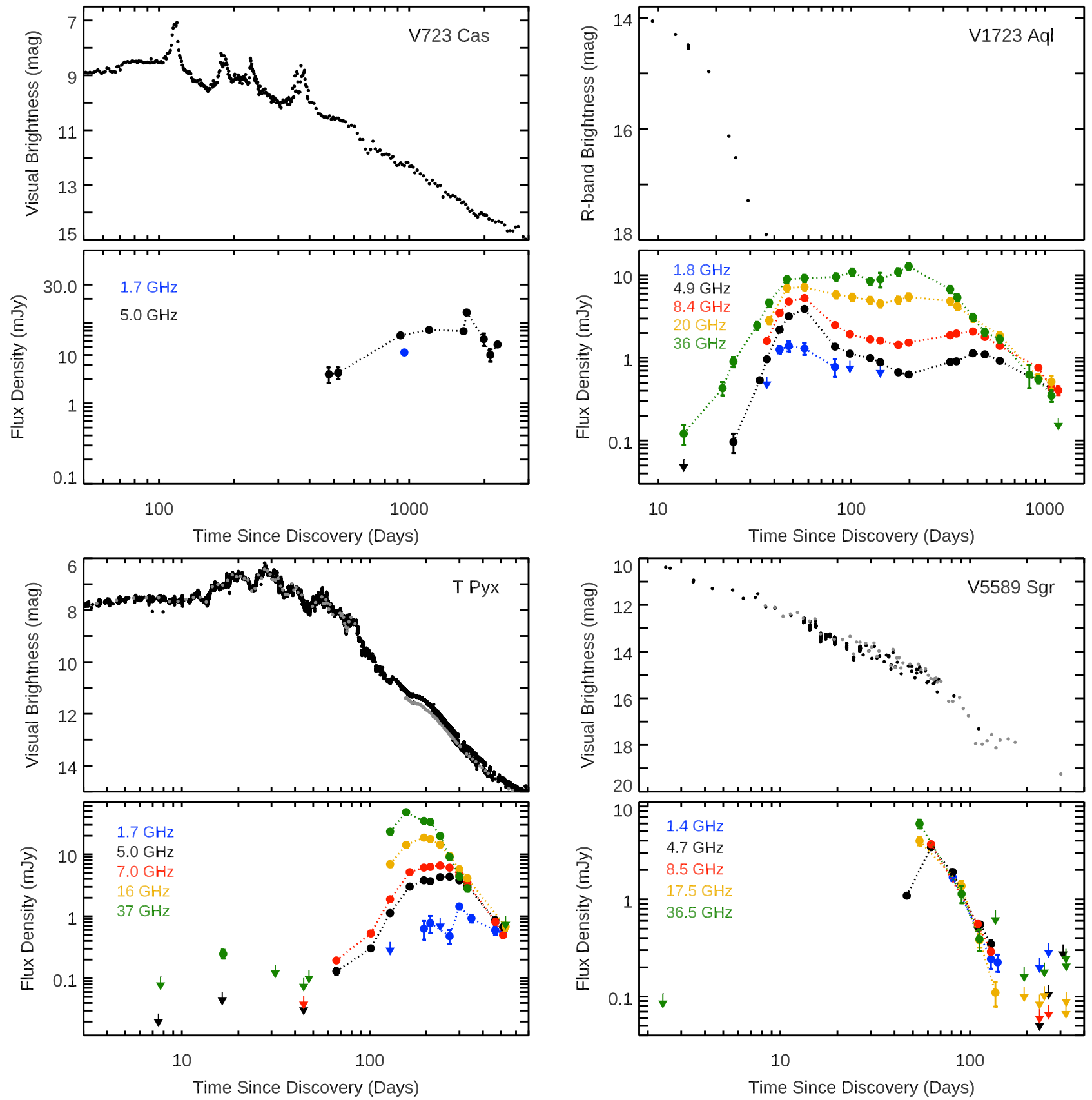

Figure 2. Previously published radio light curves, accompanied by optical light curves, for four novae (clockwise from top left): V723 Cas (1995), V1723 Aql (2010), V5589 Sgr (2012), and T Pyx (2011). Epochs with non-detections are plotted with downward-facing arrows. Optical light curves are made using AAVSO data (black points; Kafka 2020) and the Stony Brook/SMARTS Atlas of Southern Novae (grey points; Walter et al. 2012). While most are plotted at $V$ band, V1723 Aql is plotted in $R$ band, as it suffered heavy foreground extinction and observations of its $V$-band light curve are limited.

were edited, calibrated, and imaged using standard routines in AIPS, CASA, and Difmap (Greisen 2003; McMullin et al. 2007; Shepherd 1997). A bright absolute flux calibrator doubled as the bandpass calibrator. As with historic VLA data, details of calibrators and observations are available in the NRAO archive. The flux density of the complex gain calibrator is determined by a bootstrapping comparison with the absolute flux calibrator.

VLA monitoring observations tend to be short $(\sim 5-15$ minute) snapshot observations at any particular frequency. However, thanks to the many VLA baselines, uv-coverage is typically sufficient to yield high-quality images. Imaging was performed with a Briggs robust value of 0 or 1 , and all images of novae resulted in point-like emission unless otherwise noted. Due to the monitoring nature of the observations, data were collected in all VLA configurations and in a range of conditions. Poor weather can decorrelate the signal in interferometric imaging and thereby lead to underestimates of flux-especially at higher frequencies and in more extended configurations. A single iteration of phase-only self-calibration was often carried out if there was enough flux in the image $(\geqslant 10 \mathrm{mJy})$, in an attempt to correct for this decorrelation.

Observations with the ATCA made use of the $2 \mathrm{GHz}$ $(2048 \times 1 \mathrm{MHz}$ channels) bandwidth $\mathrm{CABB}$ system (Wilson et al. 2011), with central frequencies of 34.0, 19.0, 17.0, 9.0, 5.5, and $2.1 \mathrm{GHz}$. Due to the east-west nature of the ATCA 

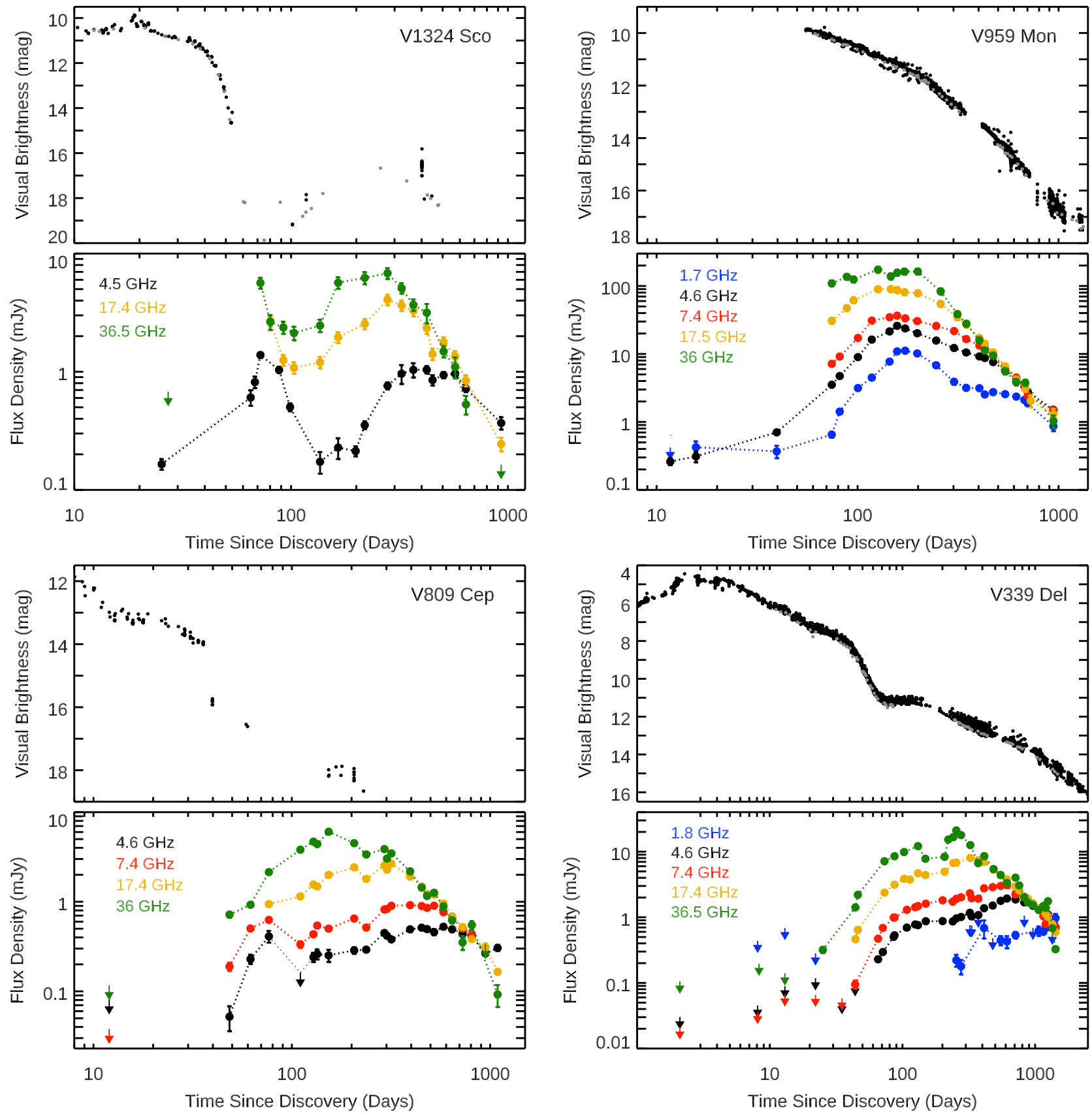

Figure 3. Previously published radio light curves, accompanied by optical light curves, for four novae (clockwise from top left): HR Del (1967), FH Ser (1970), V351 Pup (1991), and V1500 Cyg (1975). Radio epochs with nondetections are plotted with arrows. There are sporadic additional high-frequency ( $\gtrsim 15 \mathrm{GHz}$ ) points not plotted here. The visual-band light curves are binned AAVSO data published by Strope et al. (2010).

configurations, observations spanned typically $3-6 \mathrm{hr}$ to yield sufficient uv-coverage. A combination of online, automated, and manual flagging of the data was used to excise RFI; then the data were processed and analyzed using tasks within the Miriad package as outlined in the ATCA Users Guide. ${ }^{24}$ The primary flux density and bandpass calibrator was PKS B1934-638, and no self-calibration was applied. Due to the change in beam size across the $2 \mathrm{GHz}$ band, the data spanning $1.1-3.1 \mathrm{GHz}$ were divided into four subbands centered on 1.33 , $1.84,2.35$, and $2.87 \mathrm{GHz}$ prior to imaging. For the imaging, a

${ }^{24}$ https://www.narrabri.atnf.csiro.au/observing/users_guide/html/atug.html robust weighting scheme with robustness parameter $=0.5$ was used, and the image was cleaned down to a level approaching the theoretical noise.

For lower-frequency observations $(<10 \mathrm{GHz})$, we assumed a calibration error of $5 \%$, while for higher-frequency observations $(>10 \mathrm{GHz})$, we assumed $10 \%$ calibration errors. In addition, there were some epochs where no flux calibrator was observed, and we used estimates of the phase calibrator's flux density from adjacent epochs. In these cases, we imposed an additional 5\% calibration error. These calibration errors were ignored in determining which measurements were detections (significant at $>3 \sigma$ level) but are quoted in Table 3 as part of the uncertainty in flux density measurements. In cases of 

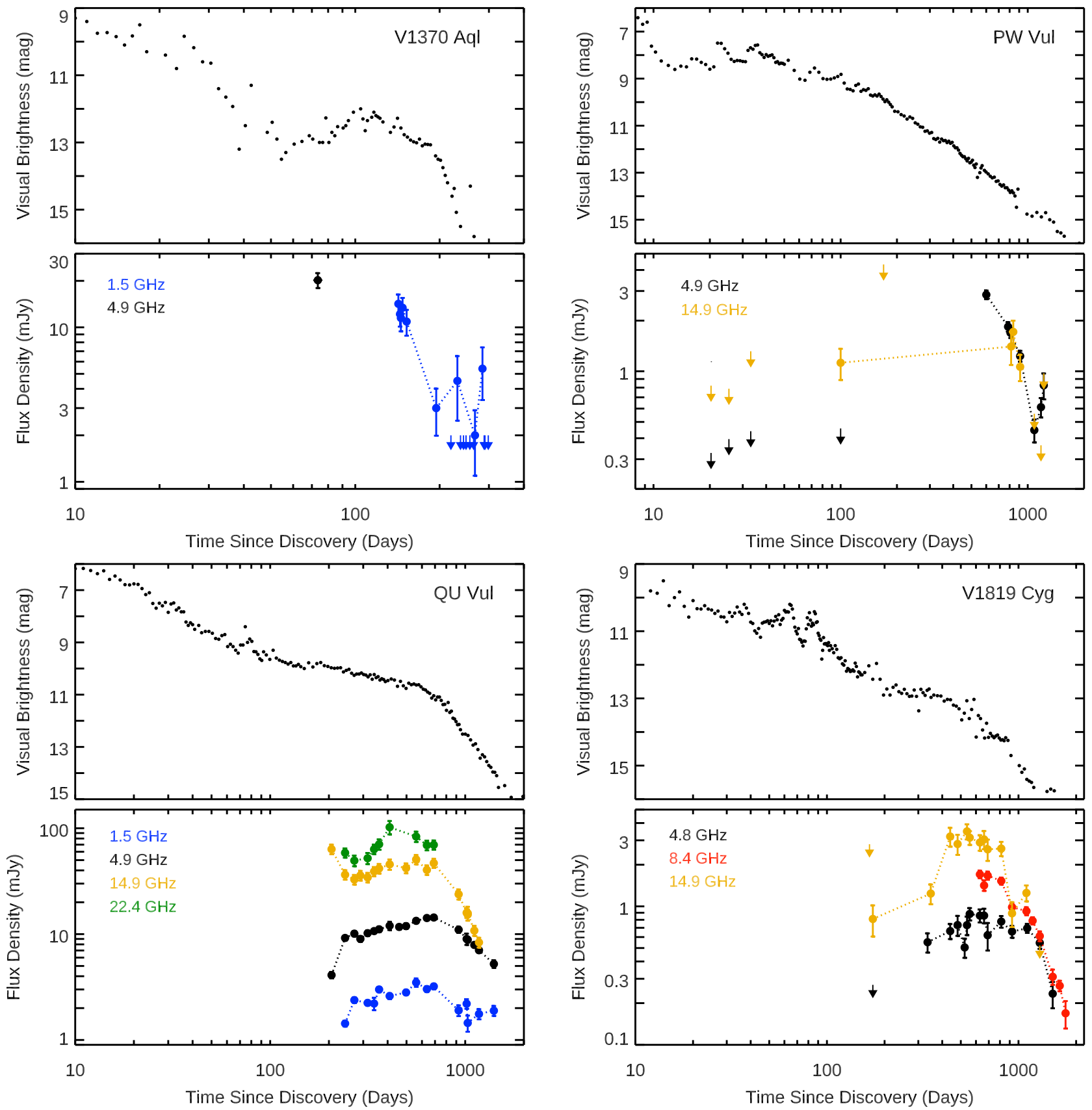

Figure 4. Optical and radio light curves for four novae (clockwise from top left): V1370 Aql (1982), PW Vul (1984), V1819 Cyg (1986), and QU Vul (1984). For radio epochs with nondetections, $3 \sigma$ upper limits are plotted with arrows; there are additional nondetections not plotted here, for clarity. The visual-band light curves are binned AAVSO data published by Strope et al. (2010).

nondetection, upper limits on the flux density are estimated by adding three times the rms background noise to the measured flux at the nova position (if it is positive; if a negatively valued noise trough is observed at the nova position, then we just take three times the image noise).

The radio flux measurements for all novae discussed in Section 2.3 are presented in the electronic version of Table 3. This table presents the observation's modified Julian date, the time elapsed between the discovery of the nova eruption and the observation $\left(t-t_{0}\right)$, the observing frequency, the flux density measurement (or $3 \sigma$ upper limit), and the $1 \sigma$ uncertainty on the flux density. The last column lists the telescope configuration; in the case of VLA observations, the array configuration (A, B, C, D, or a combination therein) is listed.

\subsection{Notes on Radio Observations of Individual Sources}

$$
\text { 2.3.1. } \mathrm{V} 1370 \mathrm{Aql}
$$

V1370 Aql was discovered on 1982 January 27 and was observed in the year following its eruption with the WSRT, primarily at $1.4 \mathrm{GHz}$ (Snijders et al. 1987). The source was revisited on 1984 May 12 by Bode et al. (1987), who observed it with the VLA at $4.9 \mathrm{GHz}$ and reported a nondetection of $<0.16 \mathrm{mJy}$. VLA observations were also obtained on 1985 June 14 (VLA program AT59; PI K. Turner) and 1987 January 

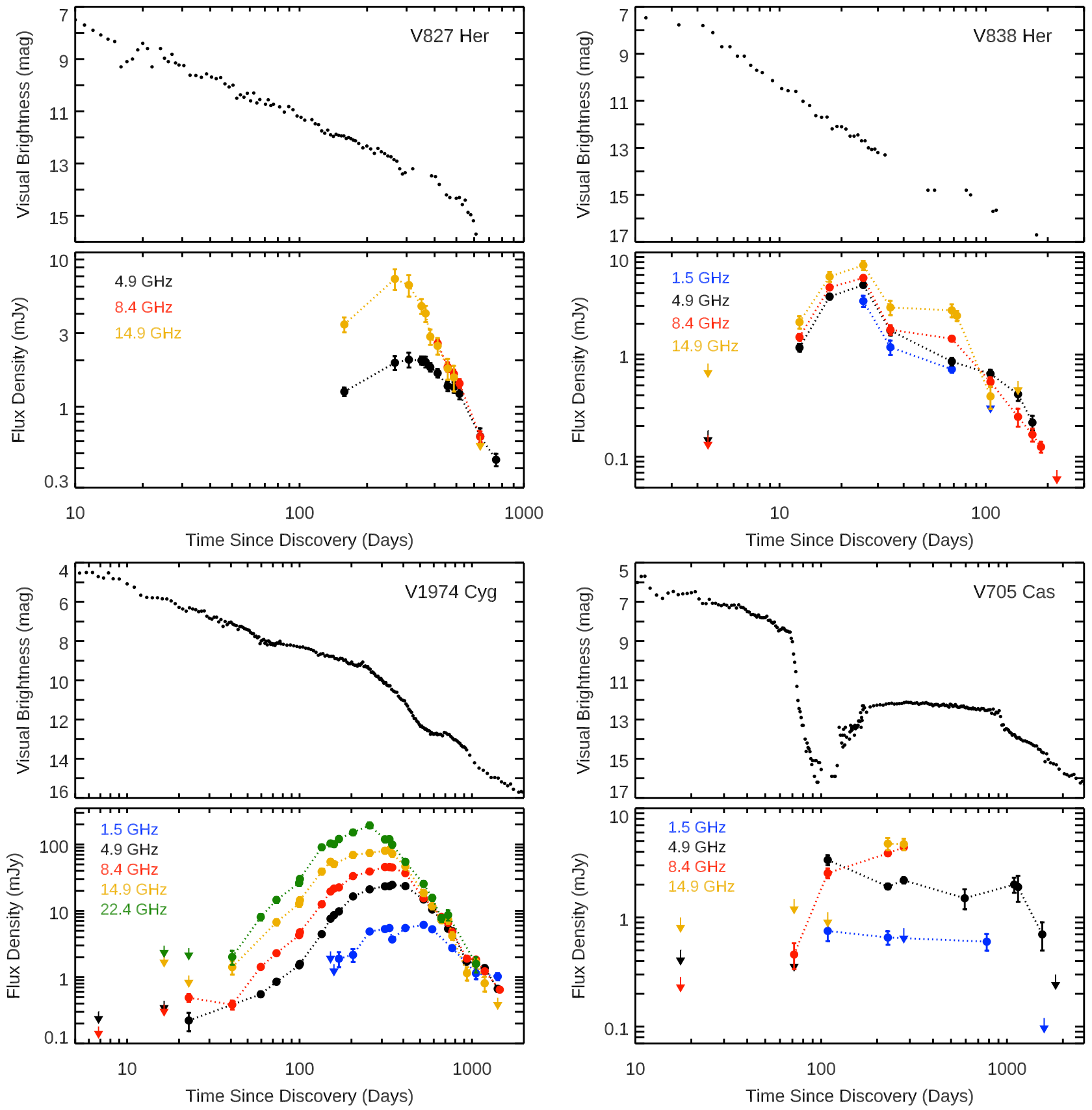

Figure 5. Optical and radio light curves for four novae (clockwise from top left): V827 Her (1987), V838 Her (1991), V705 Cas (1993), and V1974 Cyg (1992). For radio epochs with nondetections, $3 \sigma$ upper limits are plotted with arrows; there are additional nondetections not plotted here, for clarity. The visual-band light curves are binned AAVSO data published by Strope et al. (2010).

24 (VLA program AH254; PI R. Hjellming). These observations also yield nondetections. We note a nearby uncatalogued radio source at R.A. $=19^{\mathrm{h}} 23^{\mathrm{m}} 22^{\mathrm{s}} \cdot 44, \mathrm{decl} .=+2^{\circ} 29^{\prime} 04 !^{\prime \prime} 3(2000$; $28^{\prime \prime}$ from the position of V1370 Aql), which is present in all three VLA epochs with a $4.9 \mathrm{GHz}$ flux density of 1-2 mJy and which could cause some confusion if not treated carefully. The WSRT observations and VLA upper limits are plotted in Figure 4 and are listed in Table 3.

\subsection{2. $P W V u l$}

PW Vul was observed with the VLA under program IDs AH179, AH195, AH227, AH254, AH279 (PI R. Hjellming), AH185 (PI G. Hennessy), AH199 (PI E. Hummel), AT60,
AT69, and AT76 (PI A. R. Taylor). It was observed during 1984 August 17-1987 November 28, encompassing 20-1218 days after discovery. PW Vul is consistent with a point source in all epochs and frequencies, although it may be marginally resolved in A-configuration observations from 1987 July/October. Flux measurements can be found in the electronic version of Table 3 (along with values for all other novae with new data presented here), and the light curve is plotted in Figure 4.

\subsection{3. $Q U V u l$}

Radio data covering the first 500 days of eruption were published by Taylor et al. (1987). Multifrequency light curves reveal a double-peaked structure, with the first radio maximum 

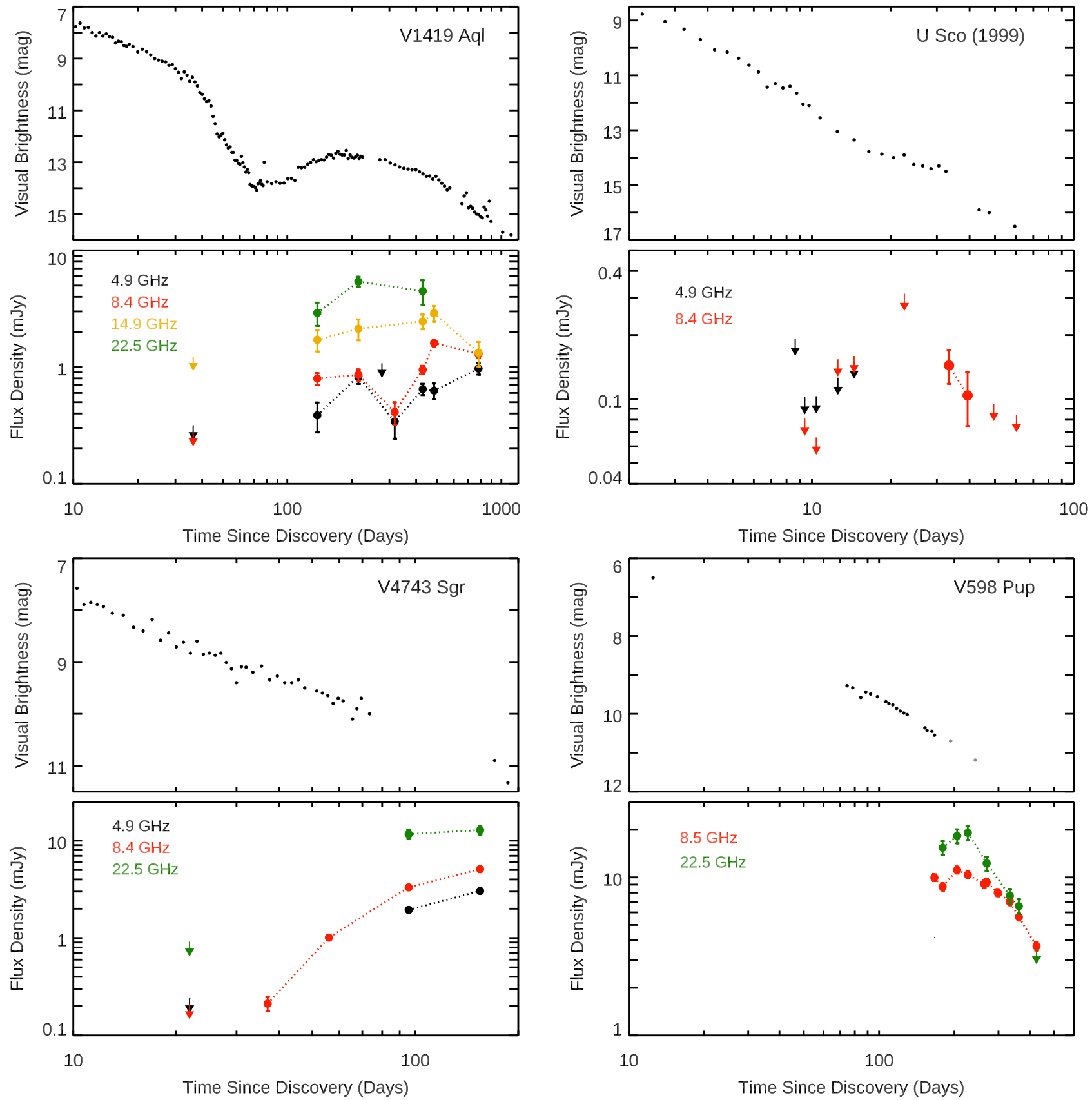

Figure 6. Optical and radio light curves for four novae (clockwise from top left): V1419 Aql (1993), U Sco (1999), V598 Pup (2007), and V4743 Sgr (2002). For epochs with nondetections, $3 \sigma$ upper limits are plotted with arrows. For the recurrent nova U Sco, we only plot data from its 1999 eruption and the first radio detections ever reported for this source; only two of its radio points are $>3 \sigma$ detections, both at $8.4 \mathrm{GHz}$. The visual-band light curves for V1419 Aql, U Sco, and V4743 Sgr are binned AAVSO data published by Strope et al. (2010), while the V598 Pup V-band light curve is from Pojmanski et al. (2007).

taking place before day 206 (and only sampled on the decline) and the second radio maximum sampled only on the rise (out to day 497). Radio monitoring of this nova continued for several more years after the publications of Taylor et al. $(1987,1988)$, and these archival data are reduced and published for the first time as part of this work (Figure 4). Overall, the light curve spans 206-1400 days after eruption, and was obtained using VLA programs AT64 (PI A.R. Taylor), AH185 (PI G. Hennessy), AH254, AH301, AH573 (PI R. Hjellming), and AJ154 (PI K. Johnston).

During the VLA's 1986 and 1987 A configurations, QU Vul was spatially resolved as discussed in Taylor et al. (1988). The next time the VLA was in A configuration, in 1988 October, QU Vul was again resolved. In fact, the nova remnant is so extended in the $14.9 \mathrm{GHz}$ image from this time that it starts to be "resolved out" (i.e., the image is very low signal-to-noise ratio $[\mathrm{S} / \mathrm{N}]$ ).

\subsubsection{V1819 Cyg}

V1819 Cyg was observed with the VLA under program IDs AH185 (PI G. Hennessy), AH254, AH279, AH301, AH316, AH366, AH390 (PI R. Hjellming), and AH385 (PI X. Han). It was observed over 1987-1991, encompassing 166-1761 days after discovery; its light curve is plotted in Figure 4. The 

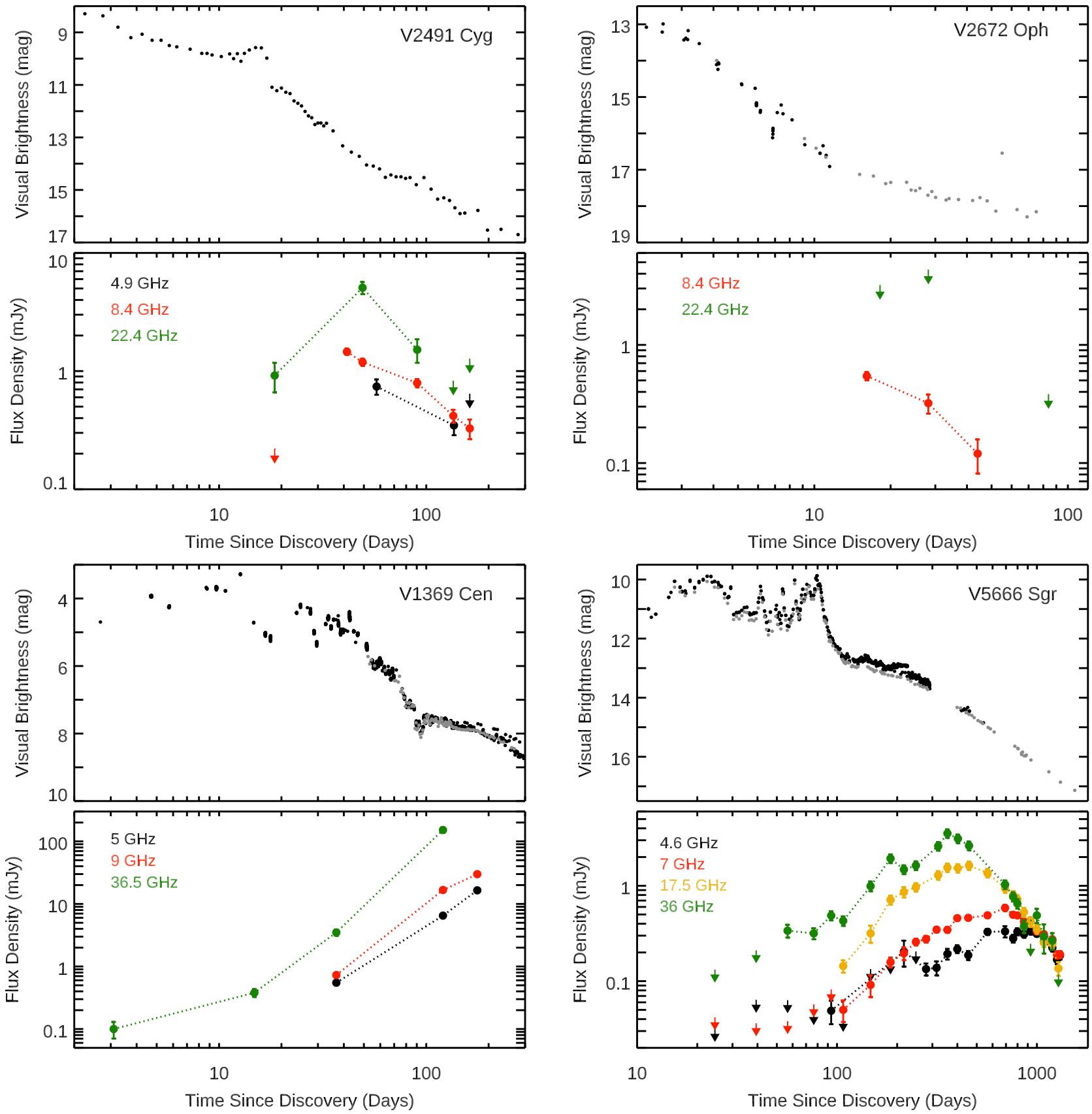

Figure 7. Optical and radio light curves for four novae (clockwise from top left): V2491 Cyg (2008), V2672 Oph (2009), V5666 Sgr (2014), and V1369 Cen (2013). For radio epochs with nondetections, $3 \sigma$ upper limits are plotted with arrows, and some nondetections are omitted for clarity. The visual-band light curve for V2491 Cyg is binned AAVSO data published by Strope et al. (2010), while we plot individual V-band AAVSO measurements (black points) and SMARTS/ Stonybrook photometry (gray points) for the other three novae.

$X$-band receivers $(8.4 \mathrm{GHz})$ were commissioned partway through the evolution of V1819 Cyg's light curve, so there are no $8.4 \mathrm{GHz}$ measurements of the early evolution. V1819 Cyg appears as a point source in all epochs and frequencies.

\subsection{5. $\mathrm{V} 827 \mathrm{Her}$}

VLA observations of V827 Her were obtained under program codes AH254, AH301, AH316 (PI R. Hjellming), and AJ154 (PI K. Johnston). Observations took place during 1987-1989, or 158-752 days after discovery, and the light curve is plotted in Figure 5. The $X$-band receivers $(8.4 \mathrm{GHz})$ were commissioned partway through the evolution of V827 Her's light curve, so there are no $8.4 \mathrm{GHz}$ measurements of the early evolution. V827 Her appears as a point source in all epochs and frequencies.

\subsection{6. $\mathrm{V} 838 \mathrm{Her}$}

VLA observations of V838 Her were obtained under program codes AB601 (PI D. A. Brown), AH390, AH428, AH444 (PI R. Hjellming), and AH424 (PI X.H. Han). Observations took place during 1991, covering 4-222 days after discovery, and are plotted in Figure 5. V838 Her appears as a point source in all epochs and frequencies. 

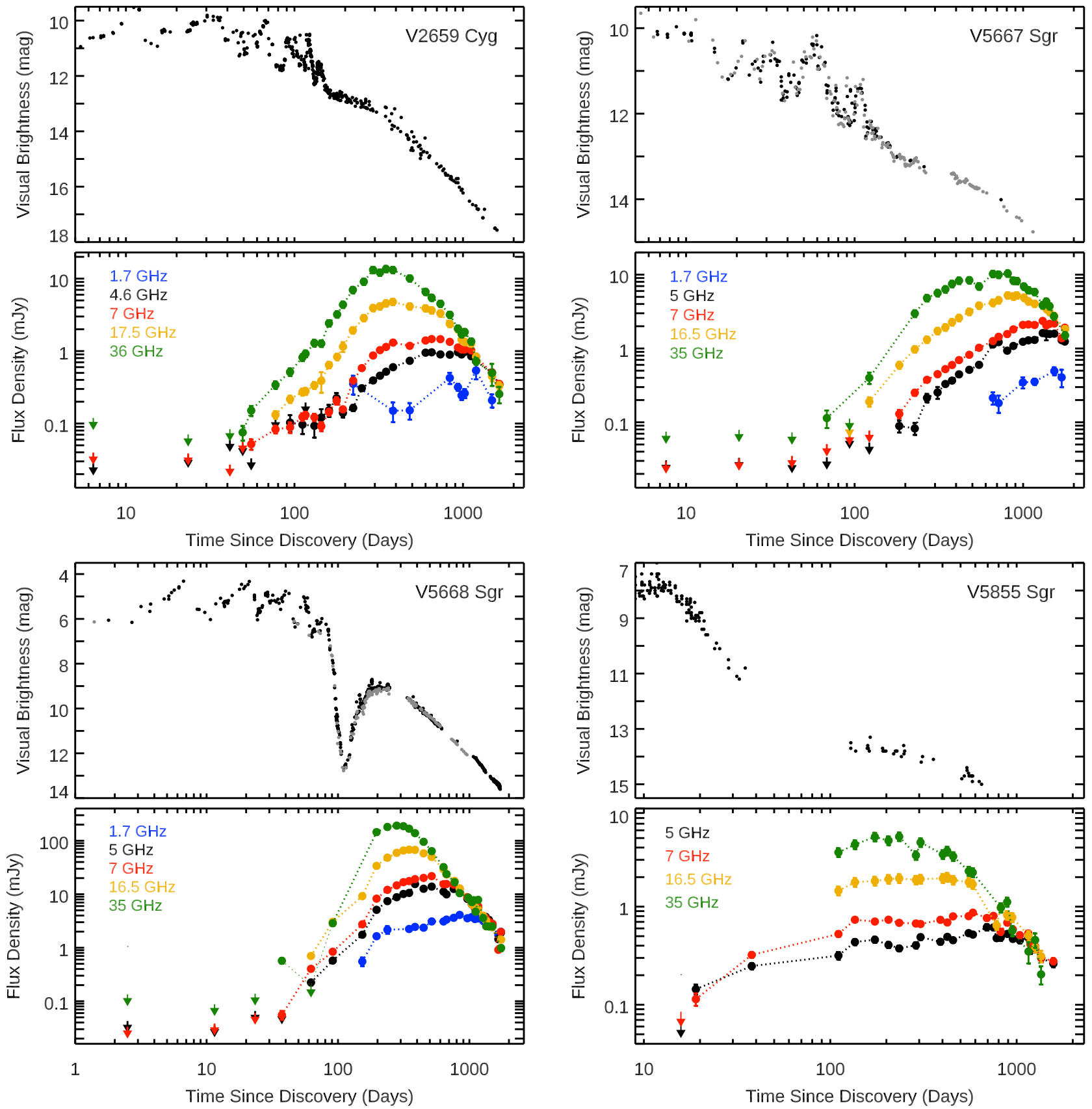

Figure 8. Optical and radio light curves for four novae (clockwise from top left): V2659 Cyg (2014), V5667 Sgr (2015), V5855 Sgr (2016), and V5668 Sgr (2015). For epochs with nondetections, $3 \sigma$ upper limits are plotted with arrows. Optical light curves are made using $V$-band or "Vis." AAVSO measurements (black points; Kafka 2020) and Stony Brook/SMARTS data (gray points; Walter et al. 2012). Note that V5668 Sgr has an early $35 \mathrm{GHz}$ detection on day 37 but then faded to a nondetection on day 62 ; these points are connected with lighter dotted lines. We also do not plot $1.7 \mathrm{GHz}$ upper limits, as their large quantity and relatively high fluxes lead to confusion with other frequencies.

\subsubsection{V1974 Cyg}

VLA observations of V1974 Cyg were tentatively presented in Hjellming (1996) but never fully cataloged in the literature, so we present them in full detail here. High-resolution MERLIN observations were presented in Pavelin et al. (1993) and Eyres et al. (1996), while monitoring at millimeter wavelengths is presented in Ivison et al. (1993).

The VLA observations were obtained under program codes AF211 (PI R. L. Fiedler), AH390, AH492, AH573 (PI R. Hjellming), and AL314 (PI E.P. Liang). Observations took place during 1992 February 25 to 1996 February 6, or 6-1448 days after discovery, and are plotted in Figure 5.

During the 1992 A configuration, V1974 Cyg was already strongly resolved at the $U$ and $K$ bands. Images were broadly consistent with a circularly symmetric ring, and total flux densities were estimated by integrating over the emitting region with AIPS/tvstat. Meanwhile, at the lower frequencies in A configuration, fluxes were determined with Gaussian fitting, but we allowed the width to vary. By the time of the 2014 A configuration, V1974 Cyg's $22.4 \mathrm{GHz}$ emission was resolved out and went nondetected. Total fluxes in the $X$ and $U$ bands 

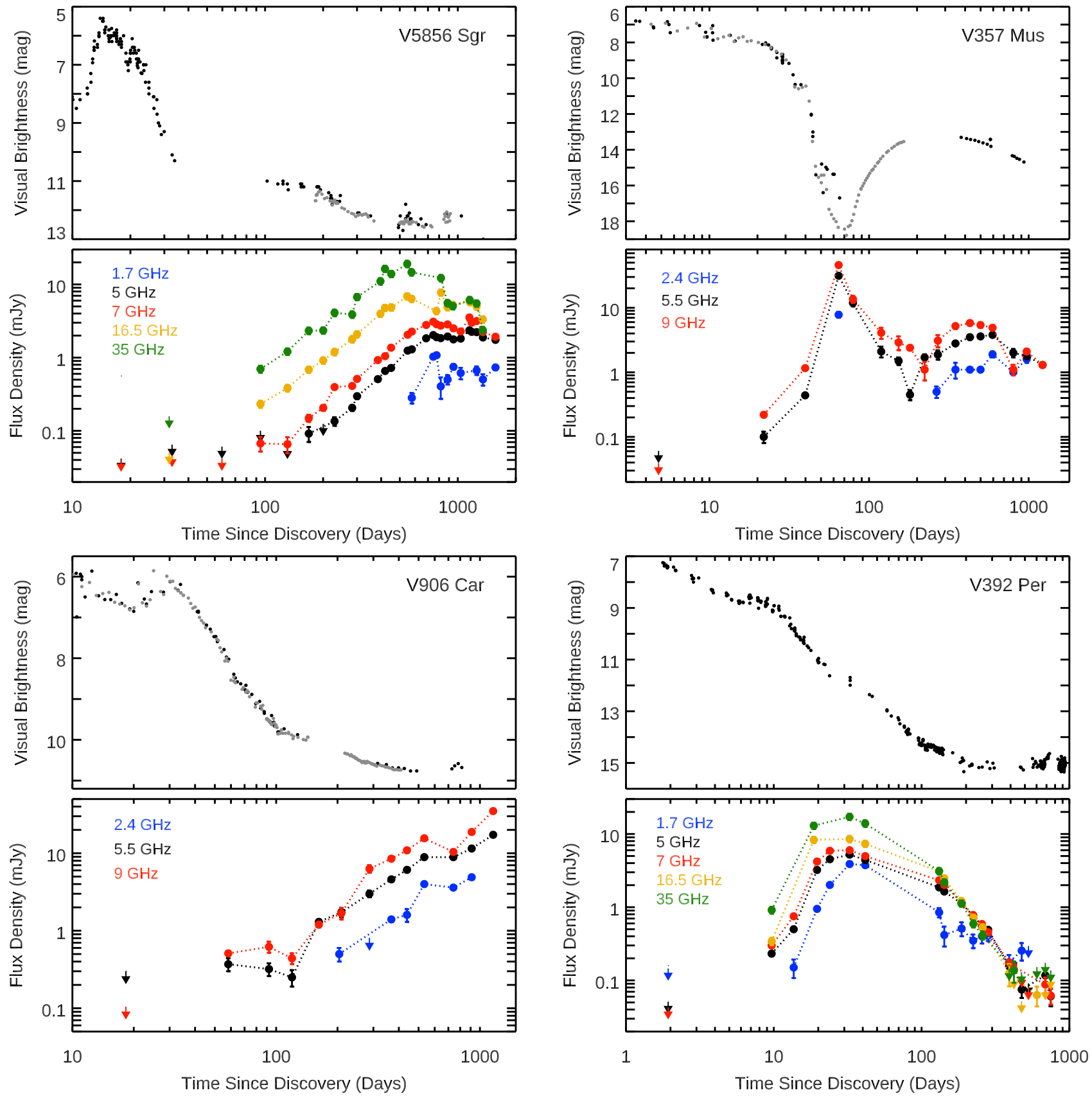

Figure 9. Optical and radio light curves for four novae (clockwise from top left): V5856 Sgr (2016), V357 Mus (2018), V392 Per (2018), and V906 Car (2018). For epochs with nondetections, $3 \sigma$ upper limits are plotted with arrows. Optical light curves are made using $V$-band or "Vis." AAVSO measurements (black points; Kafka 2020) and Stony Brook/SMARTS data (gray points; Walter et al. 2012). For V5856 Sgr, we do not plot $1.7 \mathrm{GHz}$ upper limits, as their large quantity and relatively high fluxes lead to confusion with other frequencies.

were integrated with tvstat, while Gaussian fits were performed at the $L$ and $C$ bands.

\subsubsection{V705 Cas}

VLA and MERLIN observations of the eruption of V705 Cas were presented by Eyres et al. (2000) starting on 1994 July 23 (day 228), which they claim is the first radio detection of the nova. There are several earlier epochs of VLA observations, obtained under program AH492 (PI R. Hjellming) 17-108 days after discovery, which Eyres et al. present as nondetections. We re-reduce these data here and find detections at lower frequencies ( $\leqslant 8.4 \mathrm{GHz}$ ) on 1994 February 16 (day 71) and 1994 March 25 (day 108). The flux measurements derived from these early epochs should be considered skeptically as they are afflicted by several issues, including very distant $\left(\gg 10^{\circ}\right)$ phase calibrators that may lead to data being decorrelated and flux densities being underestimated. The AH492 epoch from 1994 July 16 is unsalvageable and is omitted here, but luckily observations were obtained just 7 days later under a different program; this 1994 July 23 epoch is presented by Eyres et al. (2000). While our analysis of the $\geqslant 4.9 \mathrm{GHz}$ data is consistent with theirs, Eyres et al. report a nondetection at $1.4 \mathrm{GHz}$, while we detect the nova. Our light curve for V705 Cas is plotted in Figure 5. 
Table 3

Radio Observations of the Classical Novae in This Study

\begin{tabular}{lccccc}
\hline \hline $\begin{array}{l}\text { MJD } \\
\text { (days) }\end{array}$ & $\begin{array}{c}t-t_{0} \\
(\text { days })\end{array}$ & $\begin{array}{c}\nu \\
(\mathrm{GHz})\end{array}$ & $\begin{array}{c}\mathrm{S}_{\nu} \\
(\mathrm{mJy})\end{array}$ & $\begin{array}{c}\sigma_{S_{\nu}} \\
(\mathrm{mJy})\end{array}$ & Config \\
\hline \multicolumn{7}{c}{ V1370 Aql } \\
\hline $45,069.5$ & 73.5 & 5.00 & 20.20 & 2.24 & WSRT \\
$45,138.5$ & 142.5 & 1.41 & 14.20 & 2.12 & WSRT \\
$45,140.5$ & 144.5 & 1.41 & 12.20 & 2.09 & WSRT \\
$45,141.5$ & 145.5 & 1.41 & 11.50 & 2.08 & WSRT \\
$45,143.5$ & 147.5 & 1.41 & 13.40 & 2.11 & WSRT \\
\hline
\end{tabular}

(This table is available in its entirety in machine-readable form.)

V705 Cas was resolved by MERLIN on days 592-1548. Here we use Eyres et al.'s "visibilities" method to estimate the integrated flux densities; note that Eyres et al. (2000) warn caution in interpreting these measurements, as some flux may be resolved out by MERLIN baselines. Flux measurements from these later epochs should be treated as lower limits.

\subsection{9. $V 1419 A q l$}

VLA observations of V1419 Aql were obtained under program codes AH492 (PI R. Hjellming) and AL314 (PI E. P. Liang). Observations took place during 1991, covering 4-222 days after discovery, and are plotted in Figure 6. V1419 Aql appears as a point source in all epochs and frequencies.

\subsubsection{U Sco}

There are archival VLA data covering the 1987 and 1999 eruptions of the recurrent nova U Sco. The 1987 eruption was discovered on May 15, after light-curve maximum (Overbeek et al. 1987; Schaefer 2010). Radio observations of the 1987 eruption were obtained under programs AF138 (PI E. Fomalont) and AH185 (PI G. Hennessy) and covered 18 and 51 days after discovery, respectively. Both VLA observations from this eruption yielded nondetections.

The 1999 eruption was discovered on February 24, and peaked on 1999 February 25 (Schmeer et al. 1999; Schaefer 2010). VLA data for this eruption were obtained by PI S. Eyres as part of VLA program AE124, and covered 8-60 days after discovery. These data are published for the first time here, and remarkably, U Sco was detected at $8.4 \mathrm{GHz}$ in two VLA epochs during the 1999 eruption, on days 33 and 39 (Figure 6). These two detections are surrounded by a substantial number of nondetections, so it is clear that radio emission from U Sco is faint and fleeting. U Sco appears as a point source in the few epochs when it was detected.

The 2010 eruption of U Sco was was discovered and peaked on January 28. It was observed at longer radio wavelengths by Anupama et al. (2013), using the GMRT. They report three nondetections spanning days 1-33.

\subsubsection{1. $V 4743 \mathrm{Sgr}$}

VLA observations of V4743 Sgr were obtained under program codes AE134 (PI S. Eyres) and AI104 (PI R. Ivison) during 2002 October-2003 February, covering 21-154 days after discovery (Figure 6). V4743 Sgr appears as a point source in all epochs and frequencies.

\subsubsection{V598 Pup}

VLA observations of V598 Pup were obtained under program code AR642 (PI M. Rupen) during 2007-2008, covering 166-425 days after discovery (Figure 6). In many epochs, no standard absolute gain calibrator was observed. Instead, 0713438 was observed as the flux calibrator, which had ongoing NRAO flux monitoring on a fortnightly basis as part of their polarization calibration strategy. We do not include the $Q$-band observations here, as many of these are severely decorrelated (presumably due to atmospheric conditions), and they do not have sufficient $\mathrm{S} / \mathrm{N}$ for self calibration. V598 Pup appears as a point source in all epochs and frequencies.

\subsubsection{V2491 Cyg}

VLA observations of V2491 Cyg were obtained under program code AS946 (PI J. Sokoloski) during 2008, covering 18-162 days after discovery (Figure 7). V2491 Cyg appears as a point source in all epochs and frequencies.

\subsubsection{V2672 Oph}

VLA observations of V2672 Oph were obtained under program codes AK720 and AK722 (PI M. Krauss) during 2009, covering 16-83 days after discovery (Figure 7). V2672 Oph appears as a point source in all epochs and frequencies.

\subsubsection{V1369 Cen}

We obtained ATCA observations of the southern nova V1369 Cen under programs CX282 and VX21 (PI K. Bannister). Observations took place during the first 7 months of eruption, while the nova dramatically brightened at radio wavelengths (Figure 7). Unfortunately, due to scheduling difficulties, monitoring efforts did not continue past 2014 May for this nova. V1369 Cen appears as a point source in all epochs and frequencies.

\subsubsection{V5666 Sgr}

Jansky VLA observations of V5666 Sgr were obtained under program codes 13B-057, 16A-258 (PI L. Chomiuk), 15B-343, and 17A-335 (PI J. Linford). Observations took place during 2014 February-2017 August, covering 24-1304 days after discovery, and are plotted in Figure 7. During the 2016-2017 A configuration, V5666 Sgr appears marginally resolved in our $\mathrm{Ka}$-band observations; in these cases we let the width of the Gaussian vary and fit for the integrated flux density.

\subsubsection{V2659 Cyg}

Jansky VLA observations of V2659 Cyg were obtained under program codes 13B-057, 16A-258 (PI L. Chomiuk), 15B-343, 16B-330, 17A-335 (PI J. Linford), and 18A-415 (PI J. Sokoloski). Observations took place during 2014 April-2018 September, covering 6-1642 days after discovery, and the light curve is plotted in Figure 8.

During the 2015 A configuration, V2659 Cyg appears marginally resolved in our $\mathrm{Ka}$-band observations, and we let the width of the Gaussian vary in JMFIT and fit for the integrated flux density. In the 2016-2017 and 2018 A configurations, V2659 Cyg was resolved at $\geqslant 16.5 \mathrm{GHz}$, and 
we estimated integrated flux densities by fitting Gaussian components in the uv-plane with Difmap.

\subsubsection{8. $V 5667 \mathrm{Sgr}$}

Jansky VLA observations of V5667 Sgr were obtained under program codes S61420, 13B-057, 16A-258 (PI L. Chomiuk), 15B-343, 17A-335, 17B-313, 18B-273, 19A-298 (PI J. Linford), 18A-415 (PI J. Sokoloski), and 19B-244 (PI K. Sokolovsky). Observations took place during 2015 February2019 December, covering 7-1774 days after discovery, and are plotted in Figure 8. During the 2016 and 2018 A configurations, V5667 Sgr appears marginally resolved in our $\mathrm{Ka}$-band observations, and we let the width of the Gaussian vary and fit for the integrated flux density.

\subsubsection{9. $\mathrm{V} 5668 \mathrm{Sgr}$}

Jansky VLA observations of V5668 Sgr were obtained under program codes S61420, SA1159, 13B-057, 16A-258 (PI L. Chomiuk), 15B-343, 16B-330, 17A-335, 17B-313, 18A-365, 18B-273, 19A-298 (PI J. Linford), and 19B-244 (PI K. Sokolovsky). Observations took place during 2015 March2019 December, covering 2-1744 days after discovery, and are plotted in Figure 8.

As reported in Diaz et al. (2018), V5668 Sgr is an excellent target for interferometric imaging. It was resolved in the $\mathrm{A}$ configurations of 2016, 2018, and 2019. For our purposes here, integrated flux densities were estimated by intergrating over the emission area in Difmap. The images will be the subject of L. Takeda et al. (2021, in preparation).

\subsubsection{V5855 Sgr}

Jansky VLA observations of V5855 Sgr were obtained under program codes SA1159, 16A-318, 17B-313, 18A-365, 18B273, 19A-298, 20B-302 (PI J. Linford), 19B-244, and 20A-395, (PI K. Sokolovsky). Observations took place during 2016 November-2021 February, covering 15-1573 days after discovery, and the light curve is plotted in Figure 8. During the 2018 A configuration, V5855 Sgr appears marginally resolved in our $K a$-band observations, and we let the width of the Gaussian vary and fit for the integrated flux density.

\subsubsection{V5856 Sgr}

Jansky VLA observations of V5856 Sgr were obtained under program codes SA1159, 16A-318, 17B-313, 18A-365, 18B273, 19A-298, 20B-302 (PI J. Linford), 19B-244, and 20A395, (PI K. Sokolovsky). Observations took place during 2016 November-2021 February, covering 18-1568 days after discovery, and the light curve is plotted in Figure 9. During the 2018 A configuration, V5855 Sgr appears marginally resolved in our $\mathrm{Ka}$-band observations, and we let the width of the Gaussian vary and fit for the integrated flux density.

\subsubsection{V357 Mus}

We obtained ATCA observations of V357 Mus under programs CX371 and C3279 during 2018 January-2020 September, covering 4-972 days after discovery (Figure 9). Most epochs in the first few months of eruption only covered the $C$ band (5.5 and $9 \mathrm{GHz}$ ), with the exception of the epoch on 2018 March 19.0, when we also obtain data at $1-3 \mathrm{GHz}$ and which also just happens to be the peak of the first radio maximum. V357 Mus appears as a point source in all epochs and frequencies.

\subsubsection{V906 Car}

ATCA radio observations of $\mathrm{V} 906 \mathrm{Car}$ are published by Aydi et al. (2020a). However, that publication appeared while the radio light curve was still evolving, and we publish three additional, later epochs here (in fact, the radio light curve continues to rise up until the present day). These ATCA observations were obtained under program codes CX371 and C3279. ATCA monitoring took place during 2018 April-2020 September, covering 18-911 days after discovery, although observations continue to the present day (Figure 9). V906 Car appears as a point source in all ATCA images.

\subsubsection{V392 Per}

Jansky VLA observations of V392 Per were obtained under program codes 17B-352, 19A-298 (PI J. Linford), 19B-244, and 20A-395 (PI K. Sokolovsky). Observations took place during 2018 April-2020 May, covering 1-748 days after discovery, and the light curve is plotted in Figure 9. During the 2018 A configuration, V392 Per is unresolved. During the 2019 A configuration, V392 Per was undetected at higher frequencies and consistent with a low $\mathrm{S} / \mathrm{N}$ point source at lower frequencies.

We note that radio observations of the eruption of V392 Per were also obtained with the Arcminute Microkelvin Imager Large Array (AMI-LA), at higher cadence than the VLA observations but only at a single frequency $(15.5 \mathrm{GHz}$; Linford et al. 2018). These observations will be the subject of a future paper exploring the source in more detail.

\subsection{Multiwavelength Nova Properties}

Some basic properties of the novae in our sample are listed in Table 1. Sources were investigated using Gaia EDR3 (Gaia Collaboration et al. 2016, 2021). If there was an unambiguous Gaia match to the nova, we took the Gaia equatorial coordinates and listed them in Table 1 . In cases with more than one potential Gaia match to the nova position, we used radio observations to hone the position and determine the Gaia counterpart. Several novae have no Gaia counterpart, and in these cases we report a position measured from radio observations (these cases are noted in Table 1).

In cases where Gaia parallaxes are measured at $\geqslant 2.5 \sigma$ significance in EDR3, distances are estimated taking the prior suggested by Schaefer (2018): $150 \mathrm{pc} / \sin (l)$, where $l$ is Galactic latitude (the prior is set to zero beyond a maximum distance of $8 \mathrm{kpc}$ ). Lower-significance Gaia measurements were carefully considered; in some cases there is essentially no useful information in Gaia EDR3, as the nova was in eruption and highly variable during Gaia data acquisition. In these cases, we take distances from Gordon et al. (2021) or other sources in the literature. In other cases, where there is some, albeit lowsignificance, Gaia constraint, we consider both the literature and Gaia estimates and take whichever is most constraining and reliable. If no superscript is listed in the distance column of Table 1, this implies that the distance is from a Gaia parallax measurement, which should be revisited after future Gaia data releases. Uncertainties correspond to roughly $1 \sigma(68 \%)$ confidence intervals. 


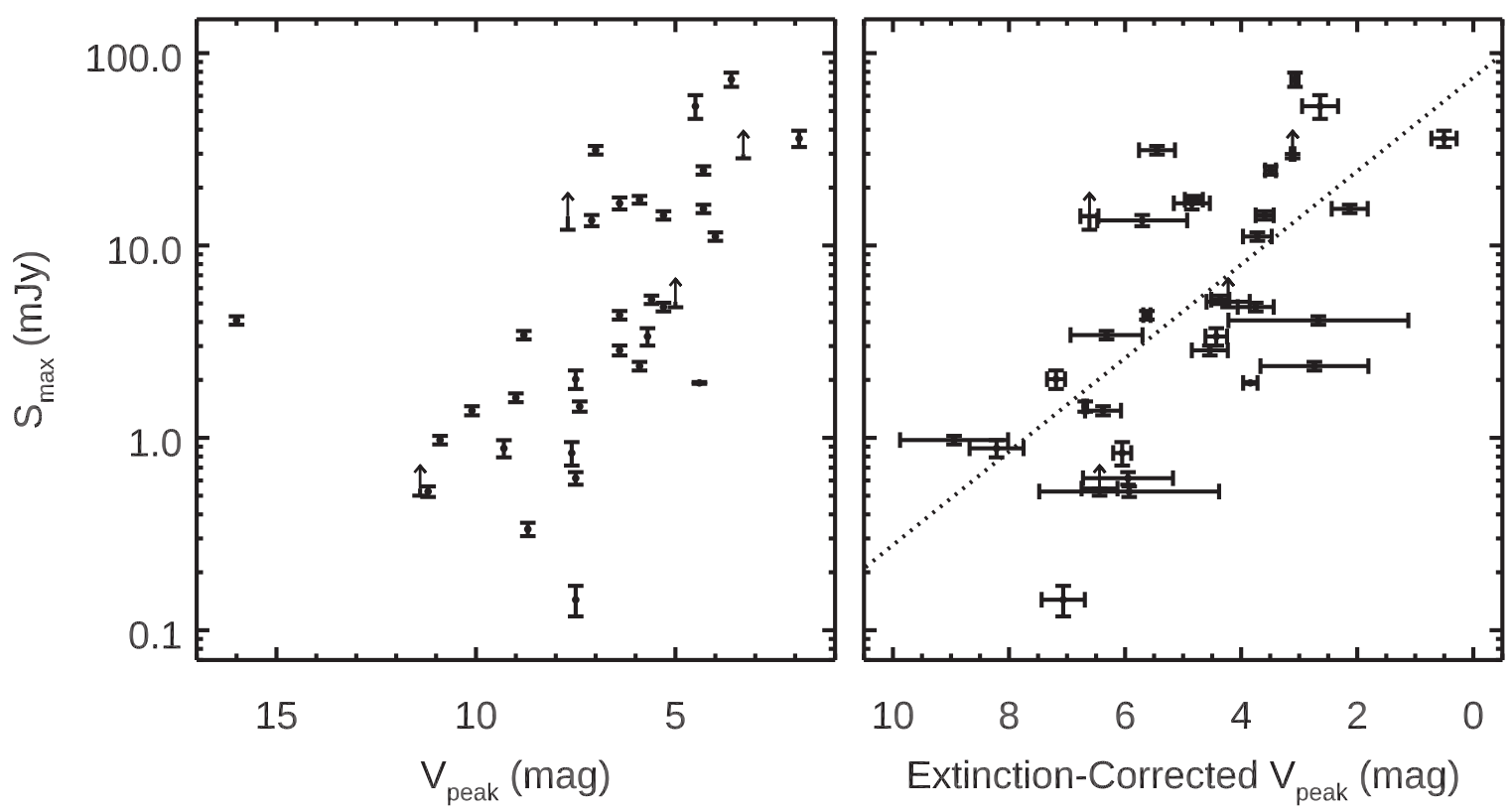

Figure 10. The flux density at radio light curve maximum $\left(S_{\max }\right)$ compared with the observed peak optical apparent magnitude $\left(V_{\text {peak }}\right.$; left panel) and the extinctioncorrected peak optical apparent magnitude (right panel). The dotted line in the right panel represents a linear fit to the data (see Section 3.1 for details). Radio flux densities are measured at $5 \mathrm{GHz}$ or, when $5 \mathrm{GHz}$ is not available, $8.5 \mathrm{GHz}$; optical brightnesses are measured in the $V$ band.
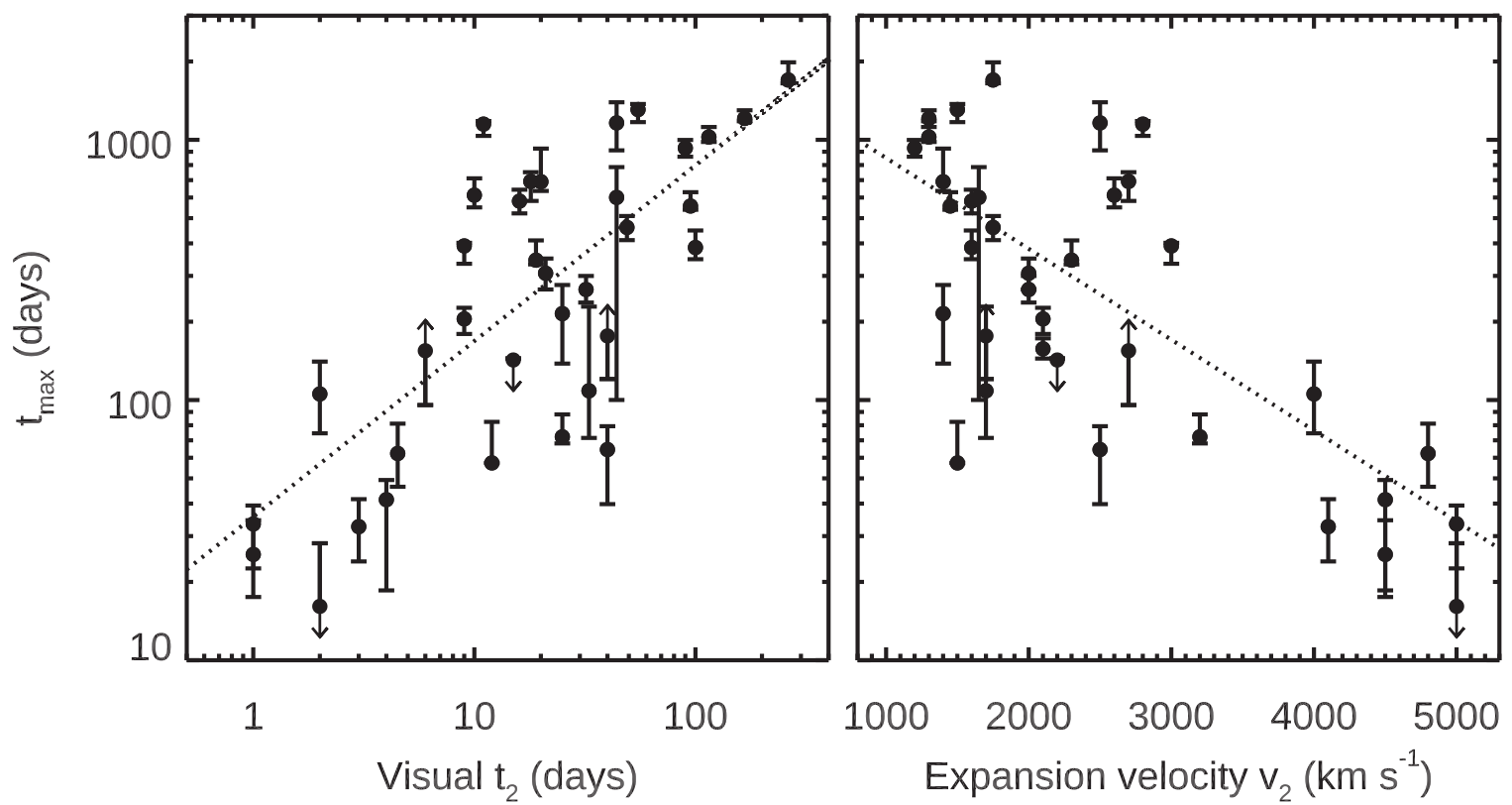

Figure 11. The time to radio light curve maximum $\left(t_{\max }\right)$ is compared, in the left panel, with the time for the optical light curve to decline by two magnitudes from peak $\left(t_{2}\right)$. In the right panel, $t_{\max }$ is compared with the expansion velocity of the ejecta $v_{2}$. The dotted lines represent linear correlations fit to the data (see Section 3.1 for details). As in Figure 10, $t_{\max }$ is measured at 5 or $8.5 \mathrm{GHz}$. The plotted uncertainties on $t_{\max }$ show the time interval between the last observation before $t_{\max }$ and the first observation after $t_{\max }$.

The date of discovery is taken as $t_{0}$, the time of start of eruption (in all cases, this is bound to be after the time of the true eruption start). All novae were discovered with optical surveys except V598 Pup and V959 Mon. V598 Pup was discovered months into eruption at X-ray wavelengths by the XMM-Newton slew survey (Saxton et al. 2008). The discovery date listed in Table 1 is actually the time V598 Pup brightened in archival optical observations obtained with the All Sky Automated Survey (Pojmanski 2002) and is remarkably well constrained to within 3 days (Read et al. 2008). V959 Mon was discovered at $\mathrm{GeV} \gamma$-ray wavelengths with the Large Area Telescope on the Fermi Gamma Ray Space Telescope while the source was in solar conjunction and was not optically identified as a nova until months later (Ackermann et al. 2014). Here we take the first $\gamma$-ray detection of V959 Mon as its time of discovery.

The optical light curves are measured in the $V$ band, and the peak observed magnitude is listed as $V_{\text {peak }}$ in Table 1 (also Figure 10). We parameterize how rapidly the optical light curve of a nova evolves with $t_{2}$, the time for the light curve to decline by two magnitudes from visual peak (Figure 11). In cases 

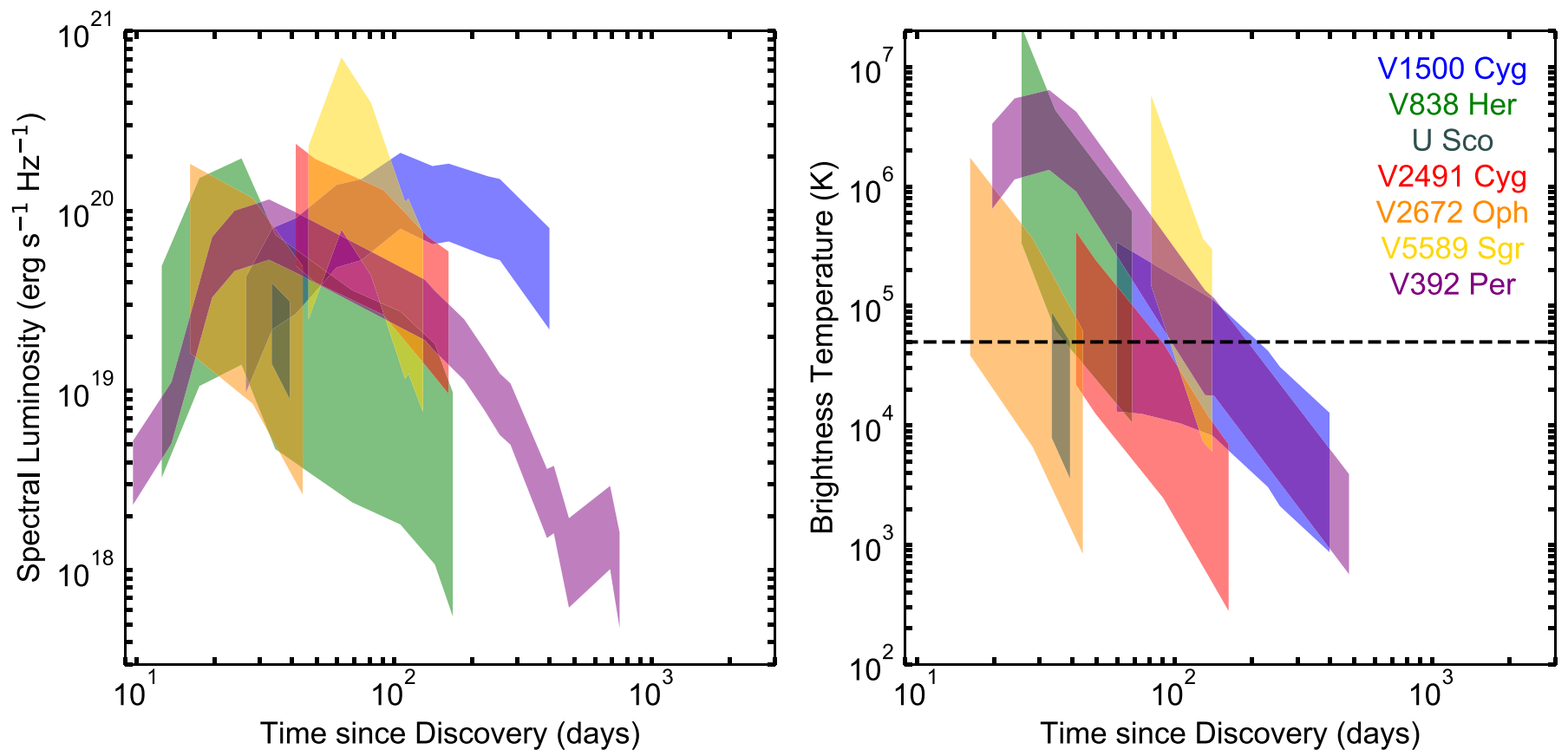

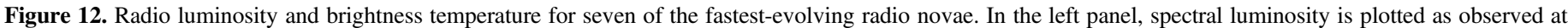

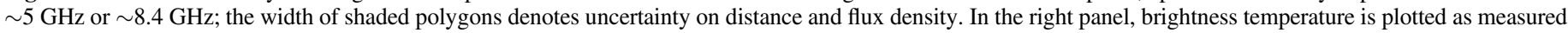

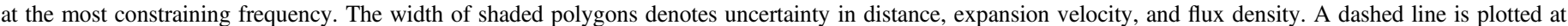
$T_{B}=5 \times 10^{4} \mathrm{~K}$ to denote the conservative maximum temperature expected for thermal emission.

where the optical light curve shows variations and "jitters," we measure $t_{2}$ as the last time the light curve crossed the $V_{\text {peak }}-2$ mag threshold, as performed by Strope et al. (2010).

Ejecta expansion velocities are estimated and interpreted within the framework put forward by Aydi et al. (2020b). The value $v_{1}$ is the velocity of the "intermediate component/ principal component" in the H Balmer lines, measured from the absorption trough of the P Cygni profiles (on top of the broad emission) a few days after optical peak; $v_{2}$ is the FWZI/ 2 of the broad $\mathrm{H}$ Balmer emission measured several days after optical peak. When spectra were available, we self-consistently measure these quantities (if no reference is provided in Table 1, velocities are measured in this current work using spectra published by, e.g., Aydi et al. 2020b). However, for older novae we are forced to estimate $v_{1}$ and $v_{2}$ from figures or values quoted in the literature. In cases where only an FWHM is available, we quote this value as $v_{2}$, as we have empirically estimated that FWZI/2 $\approx$ FWHM for typical novae around the light-curve maximum.

We obtained other parameters in Table 1 from the compilations of Ritter \& Kolb (2003), Strope et al. (2010), Özdönmez et al. (2018), and Gordon et al. (2021). In some cases, we made measurements for the first time. Light-curve parameters for V5666 Sgr, V5667 Sgr, and V2659 Cyg were determined from the light curves presented in Figure 6. The reddening for V1723 Aql is estimated from the photometry of Nagashima et al. (2013), who present a color index $V-R \approx 2.5$ around the light-curve maximum. Assuming an intrinsic color of $(V-R)_{0}=0$ around the maximum (e.g., Della Valle et al. 2002) yields reddening values of $E(V-R)=2.5$ and $E(B-V)=4.3 \mathrm{mag}$.

To constrain the emission mechanism powering nova radio luminosity (i.e., thermal or nonthermal), the brightness temperature (a measure of the radio surface brightness) is a valuable diagnostic. Brightness temperature is calculated assuming a circular disk-like emitter of diameter $\theta$ using the equation:

$$
T_{B}=1765.8 \mathrm{~K}\left(\frac{\nu}{\mathrm{GHz}}\right)^{-2} \frac{S_{\nu}}{\mathrm{mJy}}\left(\frac{\theta}{\operatorname{arcsec}}\right)^{-2} .
$$

The most reliable measurements of brightness temperature use high-resolution imaging to directly constrain the size of the emitting region and thereby the surface brightness. Unfortunately, the resolution of ATCA/VLA is not usually high enough for this to be true; instead we can approximate the angular size of the source if we know its distance, expansion velocity, and expansion time. For each nova, we use the uncertainty on distance listed in Table 1 and the range of velocities spanning $v_{1}$ to $v_{2}$ to estimate a plausible range of angular diameters $(\theta)$ at a given observation epoch. We then use this range in sizes to estimate a plausible range of brightness temperature, plotted as polygons in Figures 12-17. We note that the brightness temperatures estimated in this way are likely to be lower limits on the true brightness temperature of any nonthermal emission, as high-resolution imaging reveals that synchrotron emission is often arranged as compact knots within the more diffuse thermal ejecta (e.g., Chomiuk et al. 2014a). The brightness temperatures plotted in Figures 12-17 are for the frequency that yields the most constraining (highest) brightness temperature estimates and has reasonable temporal coverage. In most cases, this will be a relatively low frequency, often $C$ band $(\sim 5 \mathrm{GHz})$, or, when available, $L / S$ bands (1-3 GHz).

Radio luminosities are calculated assuming the distances in Table 1. The luminosities plotted in Figures 12-17 correspond to observing frequency $\sim 5 \mathrm{GHz}$ or, when that is not available, $\sim 8.5 \mathrm{GHz}$. In the case of $\mathrm{V} 1370 \mathrm{Aql}$, neither of these frequencies is available, and we instead use the $1.5 \mathrm{GHz}$ light 

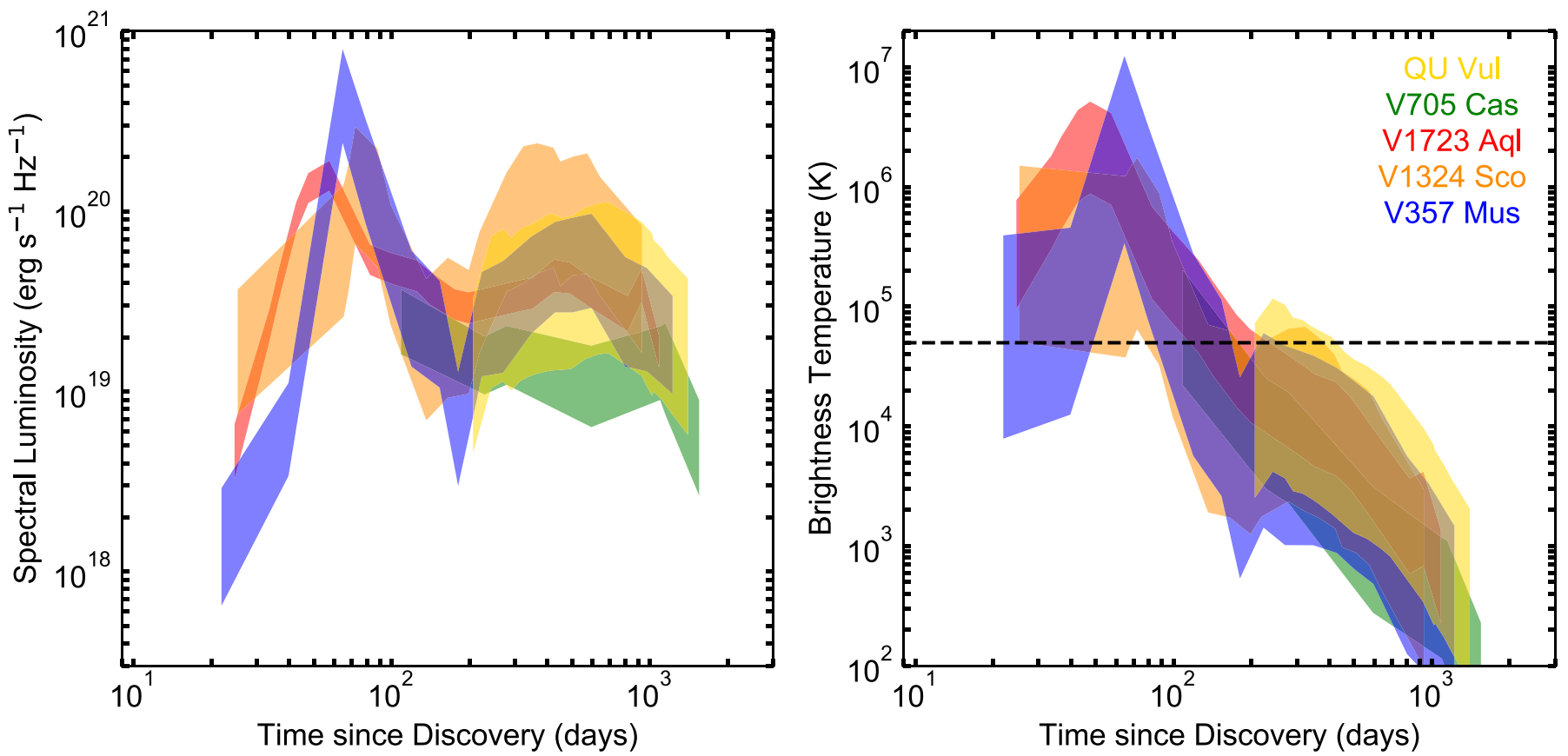

Figure 13. As in Figure 12, but here we plot five novae that show double-peaked radio light curves.
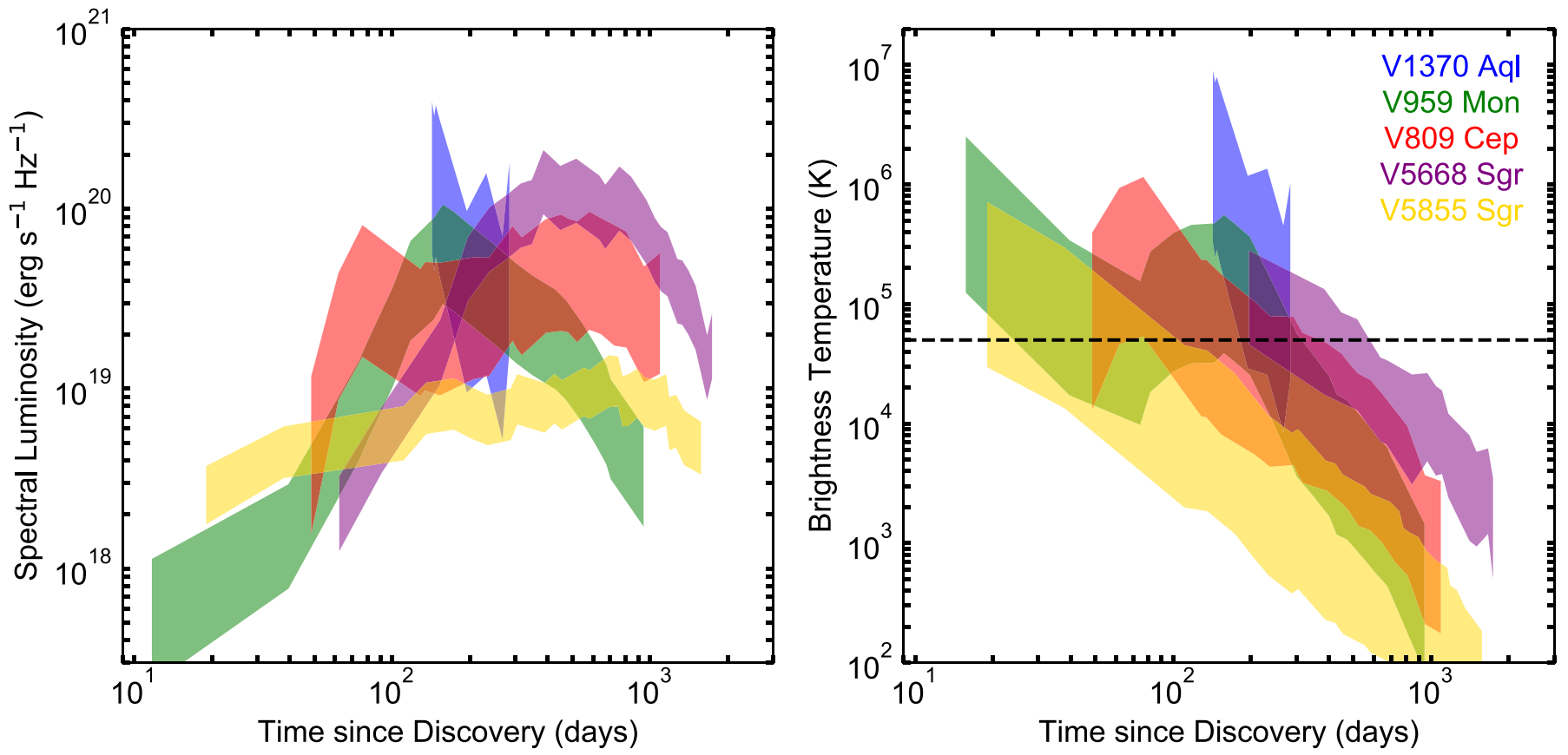

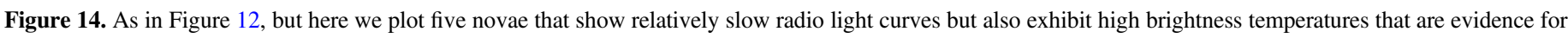
synchrotron emission.

curve. The plotted polygons denote the uncertainty band in luminosity, given measurement uncertainties on flux density and the uncertainties on distance listed in Table 1.

\section{Discussion}

The radio light curves of novae are strikingly diverse. Some remain undetected (see the Appendix), while others become some of the brightest transients in the radio sky (at least at high frequencies; e.g., V1974 Cyg). Some remain bright for a few weeks (e.g., U Sco), while others are bright for years (e.g.,
V723 Cas). Many show a single peak in their radio light curves, while others show two distinct peaks (e.g., V1723 Aql). We investigate this diversity in more detail below.

The radio spectra of novae are complex and often require more than a single power law to describe. Even when the emission mechanism is purely thermal, the radio spectrum turns over first at high frequencies, and then the turnover proceeds to lower frequencies; intermediate times can require $>2$ power laws to fully describe the spectral shape (see Nelson et al. (2014) and Weston et al. (2016a) for some examples). For these reasons, it is difficult to talk about nova spectra in terms of a 

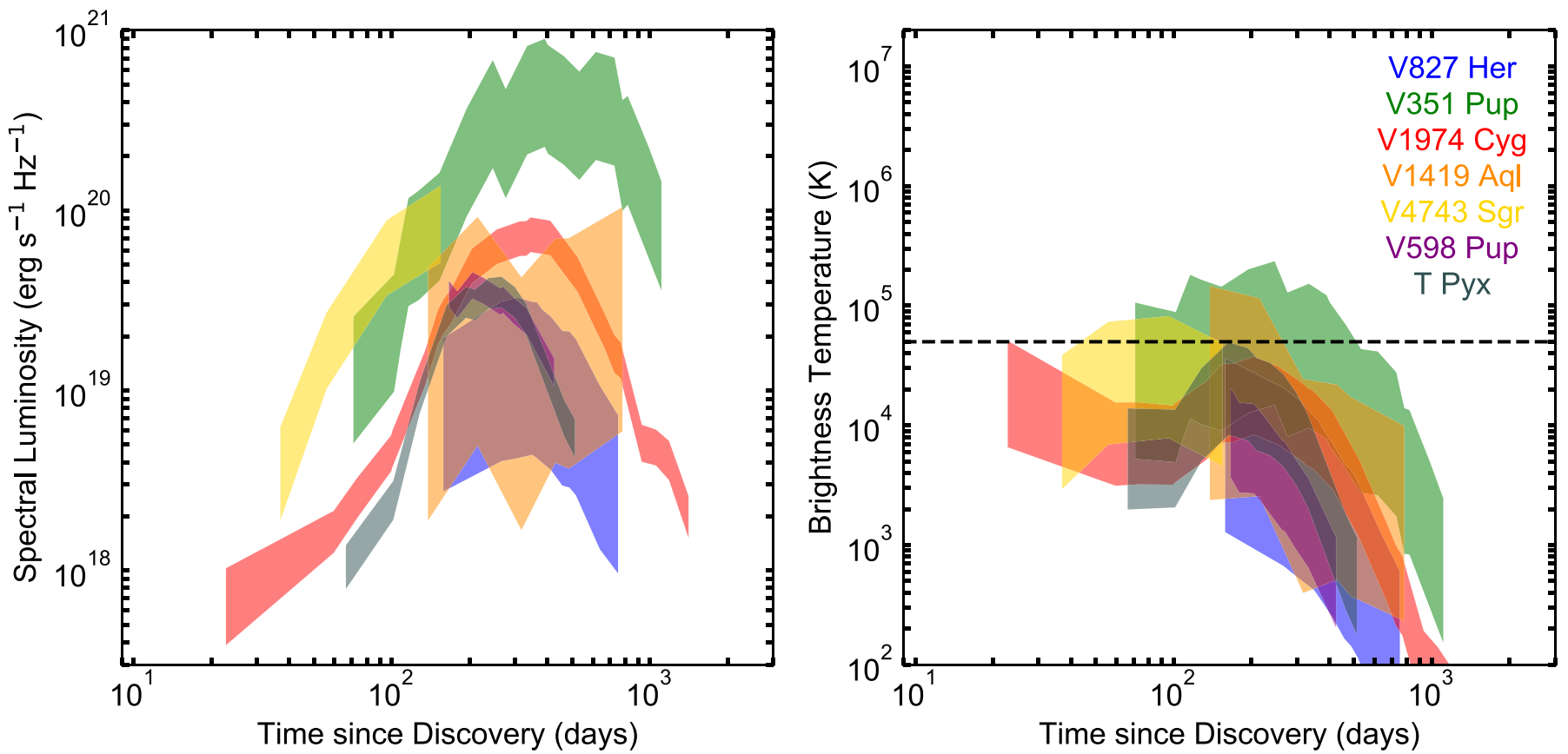

Figure 15. As in Figure 12, but here we plot seven novae that show slower evolution at radio wavelengths.
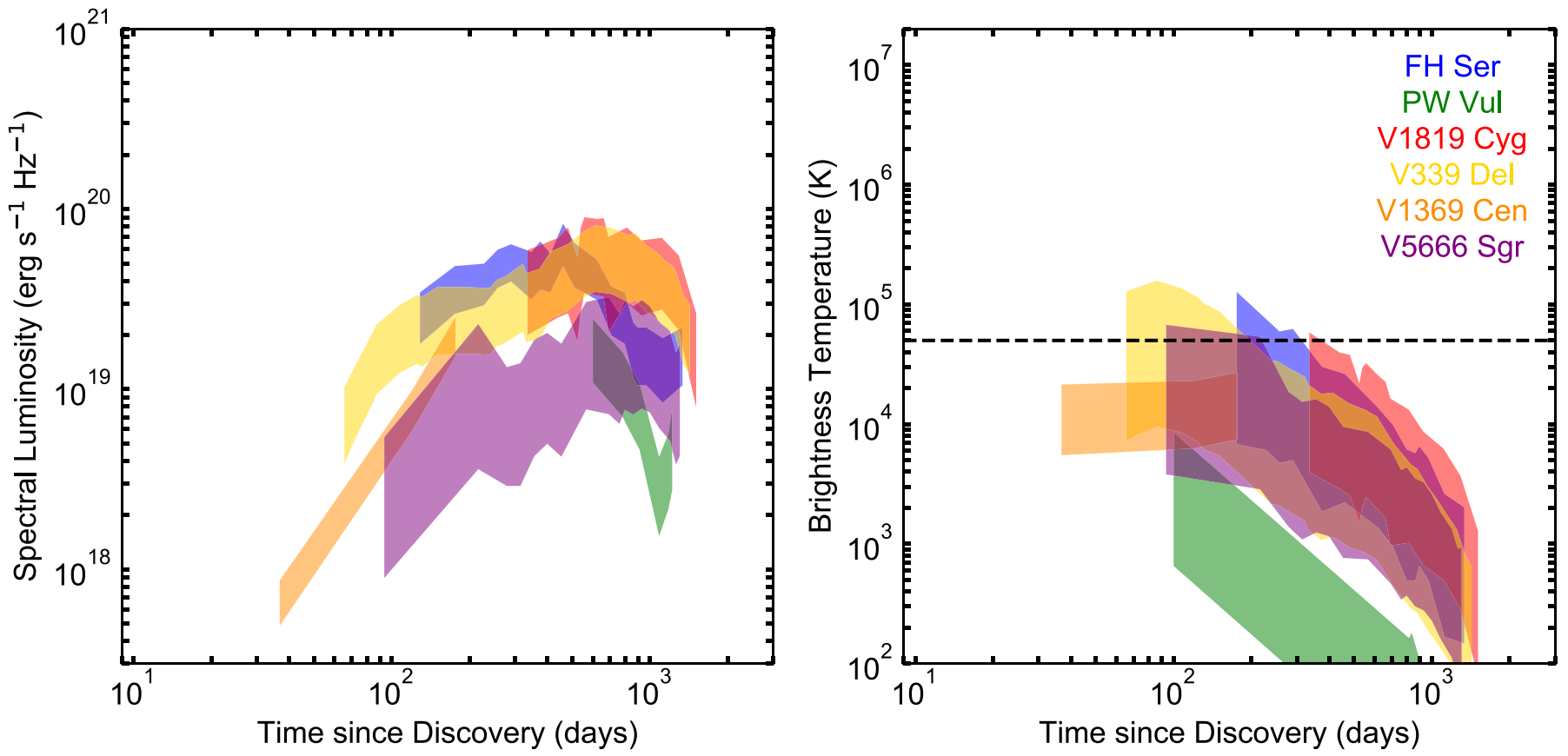

Figure 16. As in Figure 12, but here we plot six more novae that show slower evolution at radio wavelengths.

single spectral index, and discussion of the radio spectral evolution for 36 novae would rapidly become ungainly and long. We postpone discussion of radio spectral evolution to future papers, where we will investigate the thermal and synchrotron properties of these novae in more detail.

\subsection{Comparison of Basic Radio and Optical Properties}

Perhaps the diversity in radio light curves is not surprising given the range of optical light curves of novae, which show amplitudes spanning a factor of $\gtrsim 10$ magnitudes (factor of $\sim 10^{4}$ ) and $t_{2}$ spanning $>2$ orders of magnitude (Kawash et al. 2021). Both radio and optical light curves start out optically thick and transition to an optically thin state. If both optical and radio emission are thermal, powered by the warm expanding ejecta or a wind, we might expect the radio and optical light curves to track one another.

Very roughly, the peak of each light curve is set by the maximum size of the photosphere. In Figure 10, we compare the peak radio flux density ( $S_{\max }$; also listed in Table 2 ) with the peak optical magnitude. The left panel plots peak $V$-band magnitudes as observed, while the right panel corrects for 

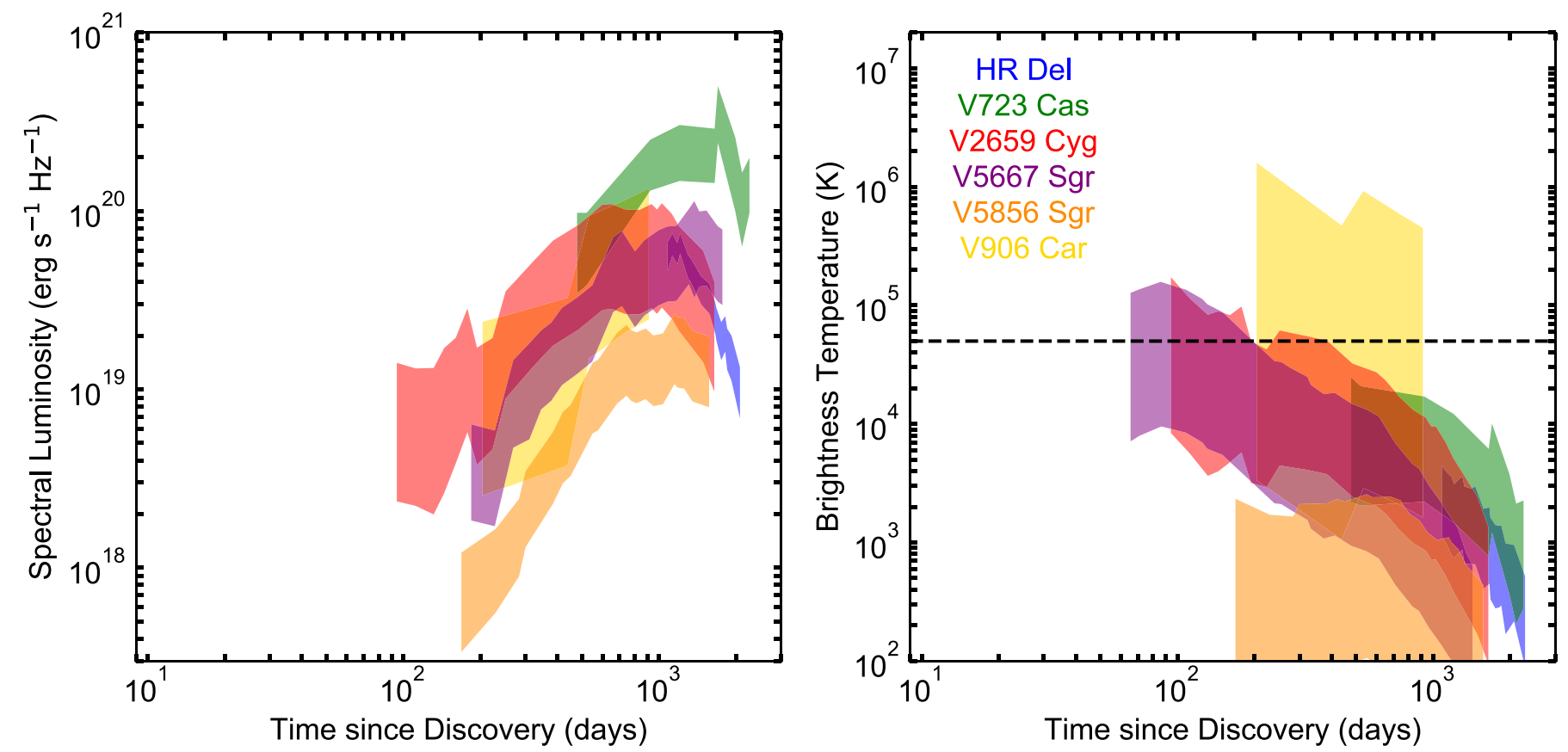

Figure 17. As in Figure 12, but here we plot six novae that show the slowest evolution at radio wavelengths.

foreground reddening using the $E(B-V)$ values tabulated in Table 1. We plot peak radio flux densities as measured at $5 \mathrm{GHz}$, or when that is not available, as measured at $8.5 \mathrm{GHz}$ (limits are estimated if the radio light curve maximum is not observed). We see that, indeed, the peak brightnesses at optical and radio wavelengths are correlated. V1723 Aql is a strong outlier in the left panel of Figure 10 because of the high Galactic extinction along its line of sight, but it falls in line with the rest of the sample in the extinction-corrected right panel.

The correlation between radio and optical peak brightness holds over three orders of magnitude in radio flux density. Fitting a linear relation to the data in the right panel of Figure $10, \quad \log \left(S_{\max }\right)=\beta\left(V_{\text {peak }, 0}-4.88\right)+\alpha$, we find $\alpha=0.69 \pm 0.09$ and $\beta=-0.24 \pm 0.05$, with a substantial scatter of $\sim 0.5 \mathrm{dex}$ around the relation $(\sigma=0.48 \pm 0.07$, to be precise). We also see that the variation in optical peak brightness is larger than in radio peak brightness (factor of $\sim 10^{4}$ compared with $10^{3}$ ), even after correcting for dust extinction. In Figures $12-17$, we see that peak radio luminosities for the majority of novae span a relatively narrow range of $\sim 10^{19}-10^{20} \mathrm{erg} \mathrm{s}^{-1} \mathrm{~Hz}^{-1}$, so much of the spread in peak radio flux density is due to distance.

We might similarly expect the timescales of the radio and optical light curves to be correlated. For example, a low-mass ejection should thin out and fade quickly at both optical and radio wavelengths. In the left panel of Figure 11, we plot the time to radio maximum $t_{\max }$ and compare it with optical $t_{2}$ (time to optical maximum usually occurs quickly after eruption and is therefore only measured for a few novae; $t_{2}$ is a more available diagnostic of light-curve speed). The novae in our sample span a factor of $\sim 300$ in optical $t_{2}$ and a factor of $\gtrsim 1000$ in radio $t_{\max }$. We see that radio and optical timescales are indeed positively correlated. A linear fit of the form $\log \left(t_{\max }\right)=$ $\beta\left(\log \left(t_{2}\right)-1.263\right)+\alpha$ returns $\alpha=2.40 \pm 0.07$ and $\beta=$ $0.68 \pm 0.11$ and is plotted as a dotted line in Figure 11. There is a scatter around this line of $\sigma=0.38 \pm 0.05$.
The right panel of Figure 11 plots the time to radio maximum against the expansion velocity $v_{2}$ (this is expected to be the speed of the faster, more spherical flow as hypothesized by Aydi et al. 2020b). Unsurprisingly, novae with faster expansion velocities also show radio light curves that evolve more quickly. We fit a line as $\log \left(t_{\max }\right)=\beta\left(v_{2} / 1000-2.51\right)+\alpha$ and found $\alpha=$ $2.40 \pm 0.07$ and $\beta=-0.35 \pm 0.06$. The scatter around this relation is $\sigma=0.40 \pm 0.05$.

\subsection{The Fastest Radio Novae: Synchrotron Flares and Thermal Blips}

While most novae have radio light curves that peak $>1 \mathrm{yr}$ after eruption, about a quarter show much faster radio light curves with time to radio maximum $\lesssim 100$ days (Figure 11 ). These fast radio novae can, in turn, be divided into two classes - those that show a later, second radio maximum (which are the subject of Section 3.3) and those that rapidly rise to maximum and then simply fade. This second class-novae that show a single rapid radio maximum-is represented here by V1500 Cyg, V838 Her, U Sco, V2491 Cyg, V2672 Oph, V5589 Sgr, and V392 Per and is plotted in Figure 12. Note that the temporal coverage on these novae varies greatly, and while in some cases like V5589 Sgr we can exclude the possibility that the nova rose to a second radio maximum, in other cases like V2491 Cyg and V2672 Oph we cannot. In fact, highercadence observations of V392 Per with AMI-LA imply that it may serve as a bridge between the sources discussed here and the double-peaked sources of Section 3.3, as a second bump is apparent in the $15.5 \mathrm{GHz}$ light curve between days 41 and 131, when the VLA was down for an electrical infrastructure upgrade (Williams et al. 2021).

The right panel of Figure 12 shows that many of these fastest novae reach brightness temperatures substantially $>10^{4} \mathrm{~K}$ at early times. In novae, brightness temperatures in excess of $5 \times 10^{4} \mathrm{~K}$ are evidence of nonthermal synchrotron emission for two reasons. The first reason is that the photoionized nova ejecta should quickly relax to electron temperatures of 
$T_{e} \approx(1-5) \times 10^{4} \mathrm{~K}$, with the value primarily determined by the white dwarf's temperature in the supersoft X-ray phase, and therefore by the white dwarf mass. The temperature should remain near this value for a time set by the ejecta mass and expansion velocity before cooling (Cunningham et al. 2015). If the gas is optically thick at a given frequency, it should show a brightness temperature equivalent to the electron temperature of the gas; as it transitions to optically thin at that frequency, the corresponding brightness temperature will drop. The implication is that, if photoionization is the only heating source in novae, radio brightness temperatures should not be $>5 \times 10^{4} \mathrm{~K}$. The second reason that brightness temperatures significantly $>10^{4} \mathrm{~K}$ imply nonthermal emission is that freefree optical depth $\propto T_{e}^{-1.35}$ (Seaquist \& Bode 2008), so it becomes harder for hotter gas to be optically thick at radio wavelengths. Therefore, while X-ray observations often imply the presence of shock-heated gas with $T_{e} \gg 10^{6} \mathrm{~K}$ (e.g., Mukai et al. 2008; Gordon et al. 2021), the hot gas would need to have very large emission measures and masses in order to be optically thick at radio wavelengths and show high radio brightness temperatures. Such high emission measures would be inconsistent with observed X-ray luminosities (Vlasov et al. 2016; see also discussions in Chomiuk et al. 2014b; Weston et al. 2016a, and Linford et al. 2017).

By this criteria, three of the novae plotted in Figure 12V838 Her, V5589 Sgr, and V392 Per-show strong evidence for synchrotron emission, with brightness temperatures $>10^{5} \mathrm{~K}$ measured in early observations. V2672 Oph is also probably dominated by nonthermal emission, although constraints on its brightness temperature are not quite strong enough to conclusively discern. V1500 Cyg, V2491 Cyg, and U Sco show brightness temperatures that could be explained with thermal emission but cannot exclude synchrotron emission.

These fastest radio novae have the highest expansion velocities in our sample, all with $v_{2} \gtrsim 4000 \mathrm{~km} \mathrm{~s}^{-1}$ (Figure 11). Such high velocities will naturally lead to more rapid radio evolution, regardless of whether the emission is thermal or nonthermal. We also expect higher-velocity shocks to transfer more energy to relativistic electrons, as the energy in relativistic particles is usually parameterized as a fraction of the postshock energy $\sim \rho v_{\mathrm{sh}}^{2}$, where $v_{\mathrm{sh}}$ is the velocity of the shock and $\rho$ is the density of gas being shocked. We might therefore expect synchrotron luminosities to be higher in novae with faster ejecta.

Evolved companions are overrepresented among this class of fastest radio novae. Of the four novae in our sample known to have orbital periods $>16 \mathrm{hr}$ and companions evolving off the main sequence (see Table 1), three of them have radio light curves that fall into this fastest category. These three novae (U Sco, V5589 Sgr, and V392 Per) are also some of the optically fastest novae in our sample (i.e., shortest $t_{2}$ ), while the fourth nova V723 Cas is one of the slowest in our sample and is included in Section 3.4. It is not surprising that novae with subgiant companions tend to have fast novae, as the evolved companion should power a high mass transfer rate that will trigger novae with relatively low ejecta masses (Yaron et al. 2005; Kalomeni et al. 2016). But notably, in at least the case of V5589 Sgr, the high brightness temperature is indicative of synchrotron emission with no evidence for a second thermal peak (contrary to what is observed for the novae in Section 3.3). This lack of a thermal peak may be attributable to low ejecta mass $\left(M_{\mathrm{ej}} \lesssim 10^{-6} M_{\odot}\right)$. As for the origin of the synchrotron emission, while we cannot exclude the possibility of shocks internal to the nova ejecta (as may be in V838 Her), such rapidly evolving nonthermal emission has primarily been seen in novae with red giant companions like V745 Sco, where the nova ejecta shock the red giant wind, accelerate particles to relativistic speeds, and create synchrotron emission (e.g., Kantharia et al. 2016). The synchrotron emission in V5589 Sgr and V392 Per may therefore be an indication of circumstellar material (CSM), presumably expelled by the evolved companion.

Naively, we do not expect significant wind CSM from a subgiant donor, which should be transferring mass via Roche lobe overflow (Webbink et al. 1983; Kalomeni et al. 2016). Still, a subgiant companion should have a higher mass transfer rate than a dwarf companion and therefore may pose more opportunity for nonconservative mass transfer and the creation of detectable CSM around the binary (intermediate between the low-density environs of cataclysmic variables and the dense CSM around symbiotic stars). To date, there is very little evidence for CSM around subgiants. As the Galactic recurrent nova with the fastest recurrence time, U Sco is one of the best studied novae and has a subgiant companion, and yet it shows $\mathrm{X}$-ray nondetections that place an upper limit on the density of the CSM (Drake \& Orlando 2010; the data presented here also do not require synchrotron emission or shocks). It is notable that V5589 Sgr and V392 Per have longer orbital periods than $\mathrm{U}$ Sco or V723 Cas, and therefore the companions are more evolved. The data presented here may be evidence of CSM density increasing as companion stars evolve across the subgiant phase and perhaps also evidence for diversity in CSM properties at a given orbital period. Clearly, radio observations of fast novae offer a unique and valuable window into the circumstellar environments of binary systems with mildly evolved donors.

Finally, we make a note of V838 Her, which shares rapid radio evolution and high brightness temperatures with V5589 Sgr and V392 Per (Figure 12) but has a dwarf donor with an orbital period of $7.1 \mathrm{hr}$ (Ingram et al. 1992; Szkody \& Ingram 1994). While we cannot rule out the possibility that V838 Her has unusually dense CSM compared with its cataclysmic variable brethren, this seems unlikely and instead may point toward internal shocks shaping its radio light curve. As typical in nova studies, V838 Her serves as a reminder for any hypothesis we present to not get too tidy! We note that the expansion velocities in V838 Her are unusually high (Table 1), which might be expected to produce more energetic shocks, regardless of whether they are internal (within the ejecta) or external (with CSM). The relative roles of internal and external shocks may be illuminated by folding in observations at other wavelengths like X-ray, which can constrain the absorbing column (e.g., Gordon et al. 2021).

\subsection{Double-peaked Radio Novae: Combination Synchrotron + Thermal Radio Transients}

Three novae-V1723 Aql, V1324 Sco, and V357 Musshow clearly double-peaked radio light curves, with the first peak occurring 50-80 days into eruption and the second peak several hundred days after eruption (Figure 13). QU Vul also shows clear indication of a similar early peak, although radio monitoring did not begin until day 206 and only the very end of the first peak was captured at $14.9 / 22.4 \mathrm{GHz}$; despite these limitations, Taylor et al. (1987) carried out an insightful 
analysis into the possible origins of the early radio maximum in QU Vul. V705 Cas is a more ambiguous case born of poorer observation quality, but it appears to show a $4.9 \mathrm{GHz}$ maximum on day 108 before plateauing at a near constant flux value for the next 1000 days (Figure 5. V809 Cep is another case that could be considered as a double-peaked radio light curve (Babul et al. 2021), but its early peak is only seen at lower frequencies (4.6/7.4 GHz; Figures 3 and 14), and the first peak is fainter than the later maximum.

V357 Mus is a never-before-published double-peaked radio nova, and by many criteria, the most dramatic. It reached a flux of $46 \mathrm{mJy}$ at $9 \mathrm{GHz}$ on day 64, which is an order of magnitude brighter than the later thermal maximum. This early peak reaches brightness temperatures $>3 \times 10^{5} \mathrm{~K}$, which is an indication that it is dominated by nonthermal emission. This first peak strikingly coincides with the timing of the dust dip in the optical light curve (Figure 9), which may be an indication of a common origin for synchrotron-emitting relativistic electrons and dust (Derdzinski et al. 2017).

The other novae in this class also show temporal coincidence between dust formation and the early nonthermal radio maximum. V1324 Sco shares many traits with V357 Mus, reaching similar brightness temperatures (Figure 13) and showing simultaneity between the first radio maximum and an optical dust dip (Figure 3). V1723 Aql was unfortunately very poorly observed in the optical, but the data that do exist imply that the fading of the optical light curve is likely due to dust production (Nagashima et al. 2013), and therefore it too may show a temporal coincidence between an optical dust dip and an early radio maximum. The brightness temperature of V1723 Aql's early radio maximum is $>9 \times 10^{5} \mathrm{~K}$ and therefore one of the more clear-cut cases for nonthermal emission (see Weston et al. 2016a for more discussion). V705 Cas shows temporal coincidence between the $4.9 \mathrm{GHz}$ maximum and the optical dust dip, but the case for a doublepeaked radio light curve is more ambiguous. We note that not all novae with dust dips in their optical light curves show double radio peaks: V5668 Sgr (Figure 8) can be described with a single radio maximum. Still, dramatic optical dust dips appear to be correlated with double-peaked radio light curves and in particular, an early synchrotron maximum. We note that asymmetry and inclination effects could be important in shaping both the optical and radio synchrotron light curves, if dust preferentially forms in, for example, the orbital plane, or if synchrotron-emitting shocks peek out from the optically thick thermal ejecta preferentially in some directions.

As discussed in Weston et al. (2016a) and Finzell et al. (2018), the second radio maxima generally have brightness temperatures $T_{B} \lesssim 10^{4} \mathrm{~K}$ and are consistent with thermal emission from the warm expanding ejecta. In future work, we plan to fit thermal light curves to all novae presented here (where possible); in cases like V357 Mus and QU Vul, this will be particularly valuable for decomposing the thermal and nonthermal contributions so that we may determine the energetics of the synchrotron emission and estimate the ejecta mass.

\subsection{Slower Radio Novae: Mostly Thermal but Deceptively Complex}

The largest group of radio light curves evolves more slowly $\left(t_{\max } \gtrsim 150\right.$ days). A handful of these slower novae also show evidence for early synchrotron emission and are collected in
Figure 14, while most are consistent with pure thermal emission and are plotted in Figures 15-17.

The novae plotted in Figure 14 further highlight the diversity of nova light curves, and in particular, how signatures of synchrotron emission can be diverse. Of the novae with evidence for nonthermal emission plotted in Figure 14, we have already discussed V809 Cep as a "bridge" object showing some evidence for two distinct radio maxima (Section 3.3). V1370 Aql has poor early time and high-frequency coverage, but it becomes surprisingly bright at $1.5 \mathrm{GHz}$, which translates to $T_{B}>10^{5} \mathrm{~K}$. V959 Mon shows an early time excess that implies high brightness temperature; this early time emission also shows a spectral index indicative of synchrotron emission and even higher brightness temperatures in the high-resolution very long baseline interferometry imaging presented by Chomiuk et al. (2014a). V5668 Sgr's radio light curve evolves relatively rapidly and shows brightness temperatures $>5 \times 10^{4}$ $\mathrm{K}$; a likely possibility is that its primary radio maximum is a mixture of synchrotron and thermal emission (and the relative proportions change with time). It is notable that V5668 Sgr is the one remaining "dust-dipper" nova in our sample that does not have a double-peaked radio light curve (Section 3.3), and it too shows significant evidence for nonthermal emission. V5855 Sgr has a peculiar radio light curve that rises quickly over $\sim 40$ days and then plateaus at a remarkably constant level (Figure 8); the rapid rise to relatively bright levels translates to $T_{B}>3 \times 10^{4} \mathrm{~K}$, which is a marginal indication of nonthermal contributions.

The remaining light curves, plotted in Figures 15-17 (grouped in order of increasing $t_{\max }$ ), are consistent with purely thermal emission from the ionized expanding ejecta, as their brightness temperatures are $\lesssim 10^{4} \mathrm{~K}$. In some cases, the temporal and frequency coverage are of sufficient quality to exclude nonthermal signatures like those seen in Figures 13-14, while in other cases observations are not sufficient to constrain the presence of, for example, an early time excess. We note that we cannot exclude nonthermal emission in these cases and encourage further studies of the radio spectrum and/or high-resolution imaging to test for its presence-but given our limited analysis here, they seem to be explained with pure thermal emission.

However, even in these cases with a single radio peak and $T_{B} \lesssim 10^{4} \mathrm{~K}$, many novae show subtle complexity in their radio light curves. Take, for example, V339 Del (Figure 3). The $4.6 \mathrm{GHz}$ light curve rises relatively quickly ( $\sim 100$ days) and then flattens out for nearly 2 years; during this "plateau" time, the slope/concavity of the light curve changes at least once. The overall impression from the light curve is that multiple emission components are contributing. Even if all emission is thermal, there is still the likely possibility of multiple components with different densities and expansion velocities, perhaps from an equatorial torus and a bipolar wind (Chomiuk et al. 2014a; Aydi et al. 2020b). Another example of potential thermal complexity is revealed by a comparison of V2659 Cyg and V5667 Sgr side by side (Figure 8). Although these light curves have similar $t_{\max }$ and $S_{\max }$, the shape of their radio peaks is quite different, with V5667 Sgr remaining bright at $35 \mathrm{GHz}$ out to $\sim 800$ days and V2659 Cyg fading much faster. A potential explanation for these different light-curve morphologies is nonspherical thermal ejecta (Ribeiro et al. 2014).

We again note that the $5 / 8.5 \mathrm{GHz}$ light curves of most novae appear to peak with radio spectral luminosities of 
$10^{19}-10^{20} \mathrm{erg} \mathrm{s}^{-1} \mathrm{~Hz}^{-1}$. Roughly, assuming $10^{4} \mathrm{~K}$ ejecta emitting free-free emission, $L_{\max } \propto M_{\mathrm{ej}}^{0.8}$ with weak dependence on the density distribution of the ejecta (Seaquist \& Bode 2008; Nelson et al. 2014). The implication is that most of these slow novae have ejecta masses in the range, $\sim 10^{-5} M_{\odot}$ to a few $\times 10^{-4} M_{\odot}$. One exception is V351 Pup, which shows an unusually high luminosity in Figure 15; a potential explanation is that the distance derived from expansion parallax by Wendeln et al. (2017) and used here may be erroneously large. Similarly, the radio luminosity of V5856 Sgr appears unusually low in Figure 17, which could be an indication of an erroneously small distance estimate (the one used here is estimated from three-dimensional reddening maps). As Gaia geometric distances become available for more and more novae, such distance ambiguities will be largely clarified, enabling study of absolute nova energetics.

\section{Conclusions}

We present multifrequency radio light curves for 36 novae with dwarf or subgiant companions, intentionally excluding novae with giant companions (but encouraging future study of their radio properties). The novae studied here span $50 \mathrm{yr}$, a range of speed classes $\left(t_{2}=1-263\right.$ days), optical brightnesses ( $V_{\text {peak }} \approx 16-2 \mathrm{mag}$ ), and optical light-curve morphologies (see Table 1 and Figures 1-9).

The large sample and high-quality modern data presented here demonstrate that novae are far from simple thermal transients; synchrotron emission and multiple emitting components prove common. Using Gaia distances and spectroscopically measured velocities, we constrain the angular size of the nova and thereby estimate brightness temperatures from our flux density measurements. Of the 36 novae presented here, nine $(25 \%)$ show evidence for nonthermal emission in the form of brightness temperatures $>5 \times 10^{4} \mathrm{~K}$ (Sections 3.2-3.4). We also expect that, on a deeper look that includes spectral analysis and/or high-resolution imaging, even more novae will exhibit definitive signatures of synchrotron emission (e.g., V959 Mon; Chomiuk et al. 2014a).

We compare radio and optical properties and find that the peak optical brightness and the peak radio brightness are positively correlated. We also find that novae with faster $t_{2}$ values and expansion speeds show shorter time to radio maximum. The time elapsed between nova eruption and radio maximum varies greatly across our targets, from $\lesssim 16$ days to 1697 days.

In a few cases, the radio emission is consistent with pure synchrotron emission (V838 Her, V5589 Sgr, and V392 Per; Figure 12); these are very rapidly evolving novae with high expansion velocities. V5589 Sgr and V392 Per also have mildly evolved companions, which may hint at a role for external shocks (between the nova ejecta and preexisting CSM) in shaping shock signatures in these systems.

Several novae show two distinct peaks in their radio light curves, with the first occurring $\lesssim 100$ days after eruption and showing $T_{B}>5 \times 10^{4} \mathrm{~K}$, and the second maximum occurring $\sim$ few years after eruption and displaying $T_{B} \lesssim 10^{4} \mathrm{~K}$ (Figure 13). Building on Metzger et al. (2014), Weston et al. (2016a), and Vlasov et al. (2016), we identify the first maximum with synchrotron emission and the second with thermal emission from the bulk of the ejecta. Novae with strong dust formation episodes are concentrated among this class of double-peaked radio novae, and there is also striking temporal coincidence between the dust dip in the optical light curve and the early synchrotron maximum. These novae therefore provide evidence for a common site of dust production and relativistic particle acceleration in novae: shocks.

Our basic analysis shows that radio emission from about half of our novae can be explained with pure thermal emissionthat is, nonthermal components can be accommodated within the data but are not required by a brightness temperature analysis (Figures 15-17). But even in these pure-thermal cases, high-quality light curves display clear complexity in their shapes, sometimes showing several changes in slope/concavity (e.g., V339 Del; Figure 3) and showing a range of durations around radio maximum (e.g., compare the light curves plotted in Figure 8). It is possible that multiple, distinct thermal components are contributing to the radio light curves. Take, for example, the popular scenario of a slowly expanding equatorial disk or torus and a faster, more symmetric wind. Depending on their relative masses, velocities, and durations, the radio emission from the two thermal components can peak at distinct times/luminosities and might explain the complex light-curve shapes observed in, for example, V339 Del.

We hypothesize that the radio emission from all novae has both thermal free-free and nonthermal synchrotron components. However, the relative luminosity, timing, and duration of the emission can vary dramatically between novae, resulting in startling diversity of radio light curves. In some novae, the thermal and synchrotron components are well spaced in time and comparable in flux (e.g., V1723 Aql, V1324 Sco; Figure 13), while in others the thermal and synchrotron contributions appear to blur together into a single radio maximum (e.g., V5668 Sgr, V5855 Sgr; Figure 14). Some novae appear dominated by synchrotron emission with little evidence for a thermal component (e.g., V838 Her, V392 Per; Figure 12), while others are consistent with pure thermal emission (Figures 15-17). In the future, we plan to study this diversity in the context of multiwavelength data in order to understand what determines the shock energetics and nonthermal luminosity of nova explosions. In particular, it remains unclear what sets the $\mathrm{GeV} \gamma$-ray luminosity of novae, which varies across at least two orders of magnitude (Franckowiak et al. 2018; Chomiuk et al. 2021), and the radio properties of novae in the Fermi era may shed light on this diversity.

The thermal properties of the radio light curves presented here should also be systematically analyzed in light of constraints on velocity, filling factor, and geometry derived from multiwavelength observations. We are embarking on such a study, which will produce the highest-quality estimates of ejecta mass for a large and diverse sample of novae, which in turn will serve as an important test of nova theory (e.g., Yaron et al. 2005).

This paper is dedicated to the memory of Bob Hjellming, in gratitude for his groundbreaking work on novae and other radio stars. We are also grateful for the help and support of NRAO staff, who were critical in obtaining these observations. We are grateful to Lars Bildsten, Bill Wolf, and Ken Shen for insightful conversations. We thank Rami Alsaberi, Aliya-Nur Babul, Perica Manojlović, and Yong Zheng for their help in data acquisition, reduction, and analysis.

LC, EA, KVS, TF, and AK are grateful for support from NSF grants AST-1751874 and AST-1907790, NASA Fermi grant 80NSSC20K1535, and a Cottrell fellowship of the 
Research Corporation. JDL acknowledges support from NASA Fermi grant 80NSSC17K0511 and the NRAO ngVLA Community Studies Program. JLS and JHSW were supported by NSF grant AST-1816100 and Heising-Simons Foundation grant No. 2017-246. JS acknowledges support from the Packard Foundation. ECK acknowledges support from the G.R.E.A.T. research environment funded by Vetenskapsrådet, the Swedish Research Council, under project No. 2016-06012, and support from the Wenner-Gren Foundations. VARMR acknowledges financial support from the Fundação para a Ciência e a Tecnologia (FCT) in the form of an exploratory project of reference IF/00498/2015/CP1302/CT0001 and from the Ministério da Ciência, Tecnologia e Ensino Superior (MCTES) through national funds and when applicable cofunded EU funds under the project UIDB/EEA/50008/2020 and supported by Enabling Green E-science for the Square Kilometre Array Research Infrastructure (ENGAGE-SKA), POCI-01-0145FEDER-022217, and PHOBOS, POCI-01-0145-FEDER029932, funded by Programa Operacional Competitividade e Internacionalização (COMPETE 2020) and FCT, Portugal.

The National Radio Astronomy Observatory is a facility of the National Science Foundation operated under cooperative agreement by Associated Universities, Inc. The Australia Telescope Compact Array is part of the Australia Telescope National Facility which is funded by the Australian Government for operation as a National Facility managed by CSIRO. We acknowledge the Gomeroi people as the traditional owners of the Observatory site. This research has made use of the International Variable Star Index (VSX) database, operated at AAVSO, Cambridge, Massachusetts, USA. We acknowledge with thanks the variable star observations from the AAVSO International Database contributed by observers worldwide and used in this research. This work has made use of data from the European Space Agency (ESA) mission Gaia (https://www. cosmos.esa.int/gaia), processed by the Gaia Data Processing and Analysis Consortium (DPAC, https://www.cosmos.esa. int/web/gaia/dpac/consortium). Funding for the DPAC has been provided by national institutions, in particular the institutions participating in the Gaia Multilateral Agreement.

Facilities: VLA, ATCA, AAVSO, Gaia.

\section{Appendix}

Some novae are observed for a few epochs at radio wavelengths, but then observations are dropped-sometimes due to nondetections, sometimes due to scheduling difficulties. The resulting coverage is not sufficient to illuminate the lightcurve evolution but could be useful for future studies, so we include these data here. While we strive to include data for all novae that were observed more than twice with the VLA (and a few that were observed even more sparsely), we cannot guarantee that all such targets are fully captured here. Flux densities for these novae are included at the end of the online version of Table 3 .

\section{OS And}

OS And was observed with the VLA in six epochs following its 1986 eruption under programs AH185 and AH254 (PI R. Hjellming). No confident detections were obtained. In Table 3, we use a discovery date of 1986 December 5.

\section{QV Vul}

QV Vul was discovered on 1987 November 15. It was observed with the VLA in five epochs following its 1987 eruption under programs AH254 and AH301 (PI R. Hjellming). No confident detections were obtained.

\section{V5587 Sgr}

V5587 Sgr was observed with the Jansky VLA in three epochs following its 2011 eruption under programs VLA/10B200 and 11A-254. As tabulated in Table 3 (with a discovery date of 2011 January 25), all observations yielded nondetections.

\section{V5588 Sgr}

Following its discovery on 2011 March 27, V5588 Sgr was observed with the Jansky VLA in six epochs under programs VLA/11A-254 and 11A-271. It shows the start of a rise at high frequency $(33 \mathrm{GHz})$, while the $5.9 \mathrm{GHz}$ observations yield mostly nondetections (the data are consistent with a marginal detection on 2011 May 15). All detections were consistent with an unresolved point source.

\section{V1312 Sco}

V1312 Sco was observed with the Jansky VLA in four epochs following its 2011 June 1 discovery under program VLA/11A-280. All observations yielded nondetections.

\section{V2676 Oph}

Following its discovery on 2012 March 25, V2676 Oph was observed with the Jansky VLA in four epochs under programs VLA/11B-170, 12A-479, and 12A-483. It shows the start of a rise at high frequency $(33 \mathrm{GHz})$, while the $5.9 \mathrm{GHz}$ observation yields a marginal detection. All detections were consistent with an unresolved point source.

\section{V2677 Oph}

V2677 Oph was observed with two Jansky VLA observations following its 2012 eruption under program VLA/12A483. It was observed on 2012 June 26.5 at the $C$ band, when it was detected with a flux density of $0.20 \pm 0.01 \mathrm{mJy}$ at $5.0 \mathrm{GHz}$ and $0.27 \pm 0.01 \mathrm{mJy}$ at $6.8 \mathrm{GHz}$. $\mathrm{Ka}$-band observations were subsequently obtained on 2012 June 28.1 at the $K a$ band, yielding a detection of $0.45 \pm 0.11 \mathrm{mJy}$ at $33.0 \mathrm{GHz}$. Both observations were obtained in the $\mathrm{B}$ configuration, and V2677 Oph appeared as a point source.

\section{V1533 Sco}

V1533 Sco was observed with the Jansky VLA in two epochs following its 2013 eruption under program VLA/13A455. The first took place on 2013 June 12.3, and the second on 2013 July 12.2; both were observed at the $C$ band and $K a$ band. All observations yielded nondetections, placing upper limits $<0.01 \mathrm{mJy}$ at $5.9 \mathrm{GHz}$ and $<0.25$ at $34 \mathrm{GHz}$.

\section{V5853 Sgr}

V5853 Sgr was observed with the Jansky VLA in three epochs following its 2016 August 6 discovery under program VLA/16A-318. As tabulated in Table 3, it shows the start of a rise at high frequency $(33 \mathrm{GHz})$, while the last $7 \mathrm{GHz}$ observation yields a marginal detection. All detections were consistent with an unresolved point source. 


\section{ORCID iDs}

Laura Chomiuk (10) https://orcid.org/0000-0002-8400-3705 Justin D. Linford (iD https://orcid.org/0000-0002-3873-5497 Elias Aydi (ib https://orcid.org/0000-0001-8525-3442 Keith W. Bannister (i) https://orcid.org/0000-0003-2149-0363 Koji Mukai ii https://orcid.org/0000-0002-8286-8094 Thomas J. Nelson (i) https://orcid.org/0000-0001-7009-2260 Stuart D. Ryder (iD https://orcid.org/0000-0003-4501-8100 Kirill V. Sokolovsky (iD https://orcid.org/0000-00015991-6863

Jay Strader (iD https://orcid.org/0000-0002-1468-9668 Miroslav D. Filipović (iD https://orcid.org/0000-00024990-9288

Tom Finzell (i) https://orcid.org/0000-0001-5948-7261 Adam Kawash (iD https://orcid.org/0000-0003-0071-1622 Erik C. Kool (ib https://orcid.org/0000-0002-7252-3877 Brian D. Metzger (1D https://orcid.org/0000-0002-4670-7509 Nirupam Roy (i) https://orcid.org/0000-0001-9829-7727 Ryan Urquhart (iD https://orcid.org/0000-0003-1814-8620 Jennifer Weston (iD https://orcid.org/0000-0001-7548-5266

\section{References}

Ackermann, M., Ajello, M., Albert, A., et al. 2014, Sci, 345, 554 Andrillat, Y., \& Houziaux, L. 1987, IAU Circ., 4388 Andrillat, Y., \& Houziaux, L. 1989, MNRAS, 238, 29P

Anupama, G. C., Kamath, U. S., Ramaprakash, A. N., et al. 2013, A\&A, 559, A121

Arkhipova, V. P., Esipov, V. F., \& Kozin, M. V. 1994, AstL, 20, 95

Aydi, E., Chomiuk, L., Izzo, L., et al. 2020b, ApJ, 905, 62

Aydi, E., Sokolovsky, K. V., Chomiuk, L., et al. 2020a, NatAs, 4, 776

Babul, A.-N., Sokoloski, J. L., Chomiuk, L., et al. 2021, arXiv:2106.15782

Balam, D., Hsiao, E. Y., \& Graham, M. 2010, IAU Circ., 9168, 1

Banerjee, D. P. K., Srivastava, M. K., Ashok, N. M., \& Venkataraman, V. 2016, MNRAS, 455, L109

Bode, M. F., \& Evans, A. 2008, Classical Novae, Vol. 43 (Cambridge: Cambridge Univ. Press)

Bode, M. F., Seaquist, E. R., \& Evans, A. 1987, MNRAS, 228, 217

Camilleri, P., McNaught, R. H., Gilmore, A. C., \& Kilmartin, P. M. 1992, IAU Circ., 5422, 1

Candy, M. P., Alcock, G. E. D., \& Zissell, R. E. 1967, IAU Circ., 2022, 1

Chochol, D., Hric, L., Urban, Z., et al. 1993, A\&A, 277, 103

Chochol, D., Shugarov, S., Katysheva, N., \& Volkov, I. 2015, in Proc. Science 255, The Golden Age of Cataclysmic Variables and Related Objects - III (Triests: Sissa Medialab), 56

Chochol, D., Shugarov, S., Pribulla, T., \& Volkov, I. 2014, CoSka, 43, 330

Chomiuk, L., Linford, J. D., Yang, J., et al. 2014a, Natur, 514, 339

Chomiuk, L., Metzger, B. D., \& Shen, K. J. 2021, ARA\&A, in press, arXiv:2011.08751

Chomiuk, L., Nelson, T., Mukai, K., et al. 2014b, ApJ, 788, 130

Collins, P., Hurst, G. M., Wils, P., et al. 1984, IAU Circ., 4023

Collins, P., Skiff, B. A., Bus, S. J., et al. 1992, IAU Circ., 5454, 1

Cunningham, T., Wolf, W. M., \& Bildsten, L. 2015, ApJ, 803, 76

Darnley, M. J., Ribeiro, V. A. R. M., Bode, M. F., Hounsell, R. A., \& Williams, R. P. 2012, ApJ, 746, 61

Della Valle, M., Pasquini, L., Daou, D., \& Williams, R. E. 2002, A\&A, 390, 155

della Valle, M., Reinsch, K., Thomas, H., \& Rampazzo, R. 1992, IAU Circ., 5427,1

Derdzinski, A. M., Metzger, B. D., \& Lazzati, D. 2017, MNRAS, 469, 1314

Diaz, M. P., Abraham, Z., Ribeiro, V. A. R. M., Beaklini, P. P. B., \& Takeda, L. 2018, MNRAS, 480, L54

Drake, J. J., \& Orlando, S. 2010, ApJL, 720, L195

Endoh, I., Soma, M., \& Nakamura, Y. 2018, CBET, 4515, 1

Eyres, S. P. S., Bode, M. F., O'Brien, T. J., Watson, S. K., \& Davis, R. J. 2000, MNRAS, 318, 1086

Eyres, S. P. S., Davis, R. J., \& Bode, M. F. 1996, MNRAS, 279, 249

Finzell, T., Chomiuk, L., Metzger, B. D., et al. 2018, ApJ, 852, 108

Finzell, T., Chomiuk, L., Munari, U., \& Walter, F. M. 2015, ApJ, 809, 160

Franckowiak, A., Jean, P., Wood, M., Cheung, C. C., \& Buson, S. 2018, A\&A, 609, A120
Furuyama, S., \& Pearce, A. 2014, CBET, 3802, 1

Gaia Collaboration, Brown, A. G. A., Vallenari, A., et al. 2021, A\&A, 649, A1

Gaia Collaboration, Prusti, T., de Bruijne, J. H. J., et al. 2016, A\&A, 595, A1 Gallagher, J. S., \& Starrfield, S. 1978, ARA\&A, 16, 171

Gallagher, J. S. I., \& Code, A. D. 1974, ApJ, 189, 303

Gordon, A. C., Aydi, E., Page, K. L., et al. 2021, ApJ, 910, 134

Greisen, E. W. 2003, in Information Handling in Astronomy - Historical Vistas., ed. A. Heck (Dordrecht: Kluwer Academic), 109

Hauschildt, P. H., Starrfield, S., Shore, S. N., et al. 1994, AJ, 108, 1008

Heywood, I., O’Brien, T. J., Eyres, S. P. S., Bode, M. F., \& Davis, R. J. 2005 , MNRAS, 362, 469

Hirayama, T., Yamamoto, M., Camilleri, P., et al. 1993, IAU Circ., 5791, 1 Hirosawa, K., Yamamoto, M., Nakano, S., et al. 1995, IAU Circ., 6213, 1

Hjellming, R. M. 1996, in ASP Conf. Ser. 93, Radio Emission from the Stars and the Sun, ed. A. R. Taylor \& J. M. Paredes (San Francisco, CA: ASP), 174

Hjellming, R. M., Wade, C. M., Vandenberg, N. R., \& Newell, R. T. 1979, AJ, 84,1619

Honda, M., Osawa, K., Osada, K., et al. 1975, IAU Circ., 2826, 1

Hutchings, J. B. 1970, PDAO, 13, 347

Hutchings, J. B., Bernard, J. E., Margetish, L., \& McCall, M. 1978, PDAO, 15,73

Iijima, T. 2006, A\&A, 451, 563

Iijima, T., \& Cassatella, A. 2010, A\&A, 516, A54

Ingram, D., Garnavich, P., Green, P., \& Szkody, P. 1992, PASP, 104, 402

Ivison, R. J., Hughes, D. H., Lloyd, H. M., Bang, M. K., \& Bode, M. F. 1993, MNRAS, 263, L43

Kafka, S. 2020, Observations from the AAVSO International Database, https://www.aavso.org

Kalomeni, B., Nelson, L., Rappaport, S., et al. 2016, ApJ, 833, 83 Kantharia, N. G., Dutta, P., Roy, N., et al. 2016, MNRAS, 456, L49 Kato, T., Hishikura, T., West, J. D., et al. 2002, IAU Circ., 7976, 2 Kaufman, R., Guido, E., \& Noschese, A. 2018, CBET, 4473, 1 Kawash, A., Chomiuk, L., Strader, J., et al. 2021, ApJ, 910, 120 Korotkiy, S., Sokolovsky, K., Brown, N. J., et al. 2012, CBET, 3089, 1

Kosai, H., Honda, M., Nishimura, S., et al. 1982, IAU Circ., 3661

Kosai, H., Sugano, M., Honda, M., Schmeer, P., \& Hurst, G. M. 1987, IAU Circ., 4307

Kosai, H., Takana, W., Watanabe, T., et al. 1984, IAU Circ., 3963 Krauss, M. I., Chomiuk, L., Rupen, M., et al. 2011, ApJL, 739, L6 Li, K.-L., Metzger, B. D., Chomiuk, L., et al. 2017, NatAs, 1, 697 Linford, J. D., Bright, J., Chomiuk, L., et al. 2018, ATel, 11647, 1 Linford, J. D., Chomiuk, L., Nelson, T., et al. 2017, ApJ, 842, 73

Linford, J. D., Ribeiro, V. A. R. M., Chomiuk, L., et al. 2015, ApJ, 805, 136 Mason, E., Shore, S. N., Drake, J., et al. 2021, A\&A, 649, A28

McMullin, J. P., Waters, B., Schiebel, D., Young, W., \& Golap, K. 2007, in ASP Conf. Ser. 376, Astronomical Data Analysis Software and Systems XVI, ed. R. A. Shaw, F. Hill, \& D. J. Bell (San Francisco, CA: ASP), 127 Metzger, B. D., Finzell, T., Vurm, I., et al. 2015, MNRAS, 450, 2739 Metzger, B. D., Hascoët, R., Vurm, I., et al. 2014, MNRAS, 442, 713 Morgan, G. E., Ringwald, F. A., \& Prigge, J. W. 2003, MNRAS, 344, 521 Mróz, P., Udalski, A., Poleski, R., et al. 2015, ApJS, 219, 26 Mukai, K., Orio, M., \& Della Valle, M. 2008, ApJ, 677, 1248 Munari, U., Moretti, S., \& Maitan, A. 2020, A\&A, 639, L10

Munari, U., Ochner, P., Dallaporta, S., et al. 2014, MNRAS, 440, 3402

Munari, U., Ribeiro, V. A. R. M., Bode, M. F., \& Saguner, T. 2011a, MNRAS, 410,525

Munari, U., Siviero, A., Dallaporta, S., et al. 2011b, NewA, 16, 209

Nagashima, M., Arai, A., Isogai, M., et al. 2013, in IAU Symp 281, Binary Paths to Type Ia Supernovae Explosions, ed. R. Di Stefano, M. Orio, \& M. Moe (Cambridge: Cambridge Univ. Press), 121

Nakano, S., Beize, J., Jin, Z. W., et al. 2008, IAU Circ., 8934, 1

Nakano, S., Kanatsu, K., Kawanishi, K., et al. 1993, IAU Circ., 5902, 1

Nakano, S., Pearce, A., \& Ayani, K. 2016, IAU Circ., 9284, 5

Nakano, S., Yamaoka, H., Itagaki, K., et al. 2009, IAU Circ., 9064, 1

Nelson, T., Chomiuk, L., Roy, N., et al. 2014, ApJ, 785, 78

Nishiyama, K., \& Kabashima, F. 2014, IAU Circ., 9271, 3

Nishiyama, K., Kabashima, F., \& Maehara, H. 2015, CBET, 4079, 1

Nyamai, M. M., Chomiuk, L., Ribeiro, V. A. R. M., et al. 2021, MNRAS, 501,1394

Overbeek, D., McNaught, R. H., Whitelock, P., Cragg, T., \& Verdenet, M. 1987, IAU Circ., 4395

Özdönmez, A., Ege, E., Güver, T., \& Ak, T. 2018, MNRAS, 476, 4162

Page, K. L., Osborne, J. P., Wagner, R. M., et al. 2013, ApJL, 768, L26

Pavana, M., Anche, R. M., Anupama, G. C., Ramaprakash, A. N., \& Selvakumar, G. 2019, A\&A, 622, A126

Pavelin, P. E., Davis, R. J., Morrison, L. V., Bode, M. F., \& Ivison, R. J. 1993, Natur, 363, 424 
Pojmanski, G. 2002, AcA, 52, 397

Pojmanski, G., Szczygiel, D., \& Pilecki, B. 2007, IAU Circ., 8899, 2 Raj, A., Munari, U., Lee, B.-C., et al. 2014, ATel, 6181, 1

Read, A. M., Saxton, R. D., Torres, M. A. P., et al. 2008, A\&A, 482, L1

Ribeiro, V. A. R. M., Chomiuk, L., Munari, U., et al. 2014, ApJ, 792, 57

Ritter, H., \& Kolb, U. 2003, A\&A, 404, 301

Rosino, L., Ciatti, F., \& della Valle, M. 1986, A\&A, 158, 34

Rosino, L., \& Iijima, T. 1987, Ap\&SS, 130, 157

Rosino, L., Iijima, T., Benetti, S., et al. 1992, A\&A, 257, 603

Rosino, L., Iijima, T., \& Ortolani, S. 1983, MNRAS, 205, 1069

Saxton, R. D., Read, A. M., Esquej, P., et al. 2008, A\&A, 480, 611

Schaefer, B. E. 2010, ApJS, 187, 275

Schaefer, B. E. 2018, MNRAS, 481, 3033

Schmeer, P., Waagen, E., Shaw, L., \& Mattiazzo, M. 1999, IAU Circ., 7113

Schwarz, G. J., Ness, J.-U., Osborne, J. P., et al. 2011, ApJS, 197, 31

Seach, J. 2015, CBET, 4080, 1

Seaquist, E. R., \& Bode, M. F. 2008, in Classical Novae, ed. M. F. Bode \& A. Evans (2nd ed.; Cambridge: Cambridge Univ. Press), 141

Seaquist, E. R., Duric, N., Israel, F. P., et al. 1980, AJ, 85, 283

Seaquist, E. R., \& Palimaka, J. 1977, ApJ, 217, 781

Seki, T., Kosai, H., Honda, M., et al. 1970, IAU Circ., 2216, 1

Shepherd, M. C. 1997, in ASP Conf. Ser. 125, Astronomical Data Analysis Software and Systems VI, ed. G. Hunt \& H. Payne (San Francisco, CA: ASP), 77

Snijders, M. A. J., Batt, T. J., Roche, P. F., et al. 1987, MNRAS, 228, 329

Stanek, K. Z., Kochanek, C. S., Brown, J. S., et al. 2016, ATel, 9669, 1
Strope, R. J., Schaefer, B. E., \& Henden, A. A. 2010, AJ, 140, 34

Sugano, M., Kosai, H., Alcock, G. E. D., et al. 1991, IAU Circ., 5222, 1

Szkody, P., \& Ingram, D. 1994, ApJ, 420, 830

Tappert, C., Vogt, N., Della Valle, M., Schmidtobreick, L., \& Ederoclite, A. 2014, MNRAS, 442, 565

Taylor, A. R., Hjellming, R. M., Seaquist, E. R., \& Gehrz, R. D. 1988, Natur, 335,235

Taylor, A. R., Pottasch, S. R., Seaquist, E. R., \& Hollis, J. M. 1987, A\&A, 183, 38

Uthas, H., Knigge, C., \& Steeghs, D. 2010, MNRAS, 409, 237

Vlasov, A., Vurm, I., \& Metzger, B. D. 2016, MNRAS, 463, 394

Waagan, E., Linnolt, M., Bolzoni, S., et al. 2011, CBET, 2700

Wakuda, M., \& Huruhata, M. 1986, IBVS, 2933, 1

Walter, F. M., Battisti, A., Towers, S. E., Bond, H. E., \& Stringfellow, G. S. 2012, PASP, 124, 1057

Webbink, R. F., Rappaport, S., \& Savonije, G. J. 1983, ApJ, 270, 678

Wendeln, C., Chomiuk, L., Finzell, T., Linford, J. D., \& Strader, J. 2017, ApJ, 840,110

Weston, J. H. S., Sokoloski, J. L., Chomiuk, L., et al. 2016b, MNRAS, 460, 2687

Weston, J. H. S., Sokoloski, J. L., Metzger, B. D., et al. 2016a, MNRAS, 457,887

Williams, M. N., Linford, J., Sokolovsky, K., et al. 2021, AAS Meeting, 53, 517.05

Wilson, W. E., Ferris, R. H., Axtens, P., et al. 2011, MNRAS, 416, 832

Yamanaka, M., Itoh, R., \& Komatsu, T. 2010, IAU Circ., 9167, 3

Yaron, O., Prialnik, D., Shara, M. M., \& Kovetz, A. 2005, ApJ, 623, 398 